\title{
Predictions for neutrinoless double-beta decay in the $3+1$ sterile neutrino scenario
}

\author{
C. Giunti ${ }^{a, b}$ and E.M. Zavanin ${ }^{a, b, c}$ \\ ${ }^{a}$ INFN, Sezione di Torino, \\ Via P. Giuria 1, I-10125 Torino, Italy \\ ${ }^{b}$ Department of Physics, University of Torino, \\ Via P. Giuria 1, I-10125 Torino, Italy \\ ${ }^{c}$ Instituto de Física Gleb Wataghin, Universidade Estadual de Campinas - UNICAMP, \\ Rua Sérgio Buarque de Holanda, 777, 13083-859 Campinas SP, Brazil \\ E-mail: giunti@to.infn.it, zavanin@gmail.com
}

ABSTRACT: We present accurate predictions of the effective Majorana mass $\left|m_{\beta \beta}\right|$ in neutrinoless double- $\beta$ decay in the standard case of $3 \nu$ mixing and in the case of $3+1$ neutrino mixing indicated by the reactor, Gallium and LSND anomalies. We have taken into account the uncertainties of the neutrino mixing parameters determined by oscillation experiments. It is shown that the predictions for $\left|m_{\beta \beta}\right|$ in the cases of $3 \nu$ and $3+1$ mixing are quite different, in agreement with previous discussions in the literature, and that future measurements of neutrinoless double- $\beta$ decay and of the effective light neutrino mass in $\beta$ decay or the total mass of the three lightest neutrinos in cosmological experiments may distinguish the $3 \nu$ and $3+1$ cases if the mass ordering is determined by oscillation experiments. We also present a relatively simple method to determine the minimum value of $\left|m_{\beta \beta}\right|$ in the general case of $N$-neutrino mixing.

Keywords: Neutrino Physics, Beyond Standard Model

ARXIV EPRINT: 1505.00978 


\section{Contents}

1 Introduction 1

2 Three-neutrino mixing $\quad 2$

2.1 Normal ordering 3

2.2 Inverted ordering 9

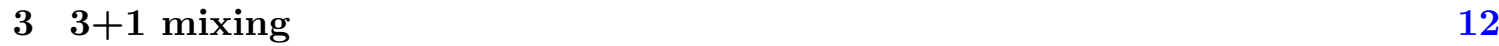

3.1 Normal ordering 13

$\begin{array}{lll}3.2 & \text { Inverted ordering } & 18\end{array}$

4 Conclusions $\quad 19$

\section{Introduction}

Neutrino flavor oscillations have been observed in many solar, reactor and accelerator experiments (see the recent reviews in refs. $[1,2]$ ), in agreement with the currently standard paradigm of three-neutrino $(3 \nu)$ mixing. The global fits of neutrino oscillation data in the framework of $3 \nu$ mixing [3-5] give us rather precise information on the values of the elements of the three mixing angles which parameterize the neutrino mixing matrix and on the values of the two independent neutrino squared-mass differences, the smaller "solar" squared-mass difference $\Delta m_{\text {SOL }}^{2} \approx 7.5 \times 10^{-5} \mathrm{eV}^{2}$ and the larger "atmospheric" squared-mass difference $\Delta m_{\mathrm{ATM}}^{2} \approx 2.4 \times 10^{-3} \mathrm{eV}^{2}$.

However, the standard $3 \nu$ mixing paradigm has been challenged by indications in favor of short-baseline oscillations generated by a new larger squared-mass difference $\Delta m_{\text {SBL }}^{2} \sim$ $1 \mathrm{eV}^{2}$ : the reactor antineutrino anomaly [6], which is a deficit of the rate of $\bar{\nu}_{e}$ observed in several short-baseline reactor neutrino experiments in comparison with that expected from the latest calculation of the reactor neutrino fluxes $[7,8]$; the Gallium neutrino anomaly [9-13], consisting in a short-baseline disappearance of $\nu_{e}$ measured in the Gallium radioactive source experiments GALLEX [14] and SAGE [15]; the signal of short-baseline $\bar{\nu}_{\mu} \rightarrow \bar{\nu}_{e}$ oscillations observed in the LSND experiment $[16,17]$. The simplest extension of $3 \nu$ mixing which can describe these short-baseline oscillations taking into account other constraints is the $3+1$ mixing scheme $[18,19]$, in which there is an additional massive neutrino at the $\mathrm{eV}$ scale and the masses of the three standard neutrinos are much smaller. Since from the LEP measurement of the invisible width of the $Z$ boson we know that there are only three active neutrinos (see ref. [20]), in the flavor basis the additional massive neutrino corresponds to a sterile neutrino [21], which does not have standard weak interactions. 
A fundamental questions that remains open is: are neutrinos Dirac or Majorana particles? This question cannot be investigated in neutrino oscillation experiments, where the total lepton number is conserved and there is no difference between Dirac neutrinos with a conserved total lepton number and truly neutral Majorana neutrinos, which do not have a conserved total lepton number. The most promising process which can reveal the Majorana nature of neutrinos is neutrinoless double-beta decay, in which the total lepton number changes by two units (see the recent review in ref. [22]).

From the present knowledge of the neutrino squared-mass differences and mixing angles it is possible to predict the possible range of values for the effective Majorana mass $\left|m_{\beta \beta}\right|$ in neutrinoless double-beta decay as a function of the absolute scale of neutrino masses (see ref. [22]), which is still unknown, up to the upper bound of about $2 \mathrm{eV}$ at $95 \%$ C.L. established by the Mainz [23] and Troitsk [24] Tritium $\beta$-decay experiments.

The introduction of a sterile neutrino at the $\mathrm{eV}$ mass scale can change dramatically the prediction for the possible range of values for the effective Majorana mass in neutrinoless double-beta decay $[13,25-33]$. In this paper we present accurate predictions for $\left|m_{\beta \beta}\right|$ taking into account the results of the global fit of solar, atmospheric and long-baseline reactor and accelerator neutrino oscillation data presented in ref. [3] and the results of an update $[34,35]$ of the global fit of short-baseline neutrino oscillation data presented in ref. [19]. We are particularly interested to determine accurately the conditions for which $\left|m_{\beta \beta}\right| \gtrsim 0.01 \mathrm{eV}$, which may be probed experimentally in the near future (see refs. [36-41]), and the conditions for which there can be a cancellation of the different mass contributions to $\left|m_{\beta \beta}\right|$, which leads to an unfortunate uncertainty for the possibility of ever observing neutrinoless double-beta decay (unless it is induced by new interactions and/or the exchange of new particles; see refs. [42-49]).

The plan of this paper is as follows. In section 2 we discuss the predictions for $\left|m_{\beta \beta}\right|$ in the standard $3 \nu$ framework, taking into account the two possible normal and inverted mass orderings. In section 3 we discuss how these predictions are modified in the $3+1$ mixing framework. In section 4 we draw our conclusions.

\section{Three-neutrino mixing}

In the standard three-neutrino $(3 \nu)$ mixing framework, the effective Majorana mass in neutrinoless double-beta decay is given by

$$
\left|m_{\beta \beta}\right|=\left|\mu_{1}+\mu_{2} e^{i \alpha_{2}}+\mu_{3} e^{i \alpha_{3}}\right|,
$$

where

$$
\mu_{k}=\left|U_{e k}\right|^{2} m_{k}
$$

is the partial contribution of the massive Majorana neutrino $\nu_{k}$ with mass $m_{k}$. The elements $U_{e k}$ of the mixing matrix, which quantify the mixing of the electron neutrino with the three massive neutrinos, can have unknown complex phases, which generate the two complex phases $\alpha_{2}$ and $\alpha_{3}$ in eq. (2.1). Since the values of these phases is completely unknown, all predictions of the value of $\left|m_{\beta \beta}\right|$ must take into account all the possible range of these phases, from 0 to $2 \pi$. 
We use the results of the global fit of solar, atmospheric and long-baseline reactor and accelerator neutrino oscillation data presented in ref. [3], which are given in terms of the mixing angles $\vartheta_{12}, \vartheta_{13}$ that determine the absolute values of the first row of the mixing matrix $U$ in the standard parameterization:

$$
\begin{aligned}
& \left|U_{e 1}\right|=\cos \vartheta_{13} \cos \vartheta_{12}, \\
& \left|U_{e 2}\right|=\cos \vartheta_{13} \sin \vartheta_{12}, \\
& \left|U_{e 3}\right|=\sin \vartheta_{13} .
\end{aligned}
$$

The results for the neutrino squared-mass differences are expressed in terms of the solar and atmospheric squared mass differences, which are defined by

$$
\begin{aligned}
& \Delta m_{\mathrm{SOL}}^{2}=\Delta m_{21}^{2}, \\
& \Delta m_{\mathrm{ATM}}^{2}=\frac{1}{2}\left|\Delta m_{31}^{2}+\Delta m_{32}^{2}\right|,
\end{aligned}
$$

where $\Delta m_{j k}^{2}=m_{j}^{2}-m_{k}^{2}$. Given this assignment of the squared mass differences, it is currently unknown if the ordering of the neutrino masses is normal (NO), such that $m_{1}<$ $m_{2}<m_{3}$ or inverted (IO), such that $m_{3}<m_{1}<m_{2}$. We discuss these two cases separately in the following subsections.

\subsection{Normal ordering}

In order to study the case of Normal Ordering (NO), we express the neutrino masses in terms of the lightest neutrino mass $m_{\min }$ :

$$
\begin{aligned}
m_{1} & =m_{\mathrm{min}}, \\
m_{2} & =\sqrt{m_{\mathrm{min}}^{2}+\Delta m_{\mathrm{SOL}}^{2}}, \\
m_{3} & =\sqrt{m_{\mathrm{min}}^{2}+\Delta m_{\mathrm{ATM}}^{2}+\Delta m_{\mathrm{SOL}}^{2} / 2} .
\end{aligned}
$$

Figure 1 shows the best-fit values and the $1 \sigma, 2 \sigma$ and $3 \sigma$ allowed intervals of the three partial mass contributions to $\left|m_{\beta \beta}\right|$ in eq. (2.1) as functions of the lightest mass $m_{1}$. We calculated the confidence intervals using the $\chi^{2}$ function

$$
\chi_{3 \nu}^{2}=\chi^{2}\left(\Delta m_{\mathrm{SOL}}^{2}\right)+\chi^{2}\left(\Delta m_{\mathrm{ATM}}^{2}\right)+\chi^{2}\left(\sin ^{2} \vartheta_{12}\right)+\chi^{2}\left(\sin ^{2} \vartheta_{13}\right),
$$

with the partial $\chi^{2}$ 's extracted from figure 3 of ref. [3], neglecting possible small correlations of the four mixing parameters. ${ }^{1}$ For each value of $m_{1}$ we calculated the confidence intervals for one degree of freedom. Although this method is in principle better than the method based on the propagation of errors of the parameters used by many authors, in practice it leads to similar results, because the $\chi^{2}$ 's of $\Delta m_{\mathrm{SOL}}^{2}, \Delta m_{\mathrm{ATM}}^{2}, \sin ^{2} \vartheta_{12}$ and $\sin ^{2} \vartheta_{13}$ in figure 3 of ref. [3] are very well approximated by quadratic functions, which correspond to Gaussian uncertainties for which the method of propagation of errors is valid.

\footnotetext{
${ }^{1}$ The only significant correlations discussed in ref. [3] are those which involve the mixing angle $\vartheta_{23}$, which is irrelevant for neutrinoless double- $\beta$ decay, and the Dirac phase, whose effect in neutrinoless double- $\beta$ decay is masked by the two unknown Majorana phases.
} 


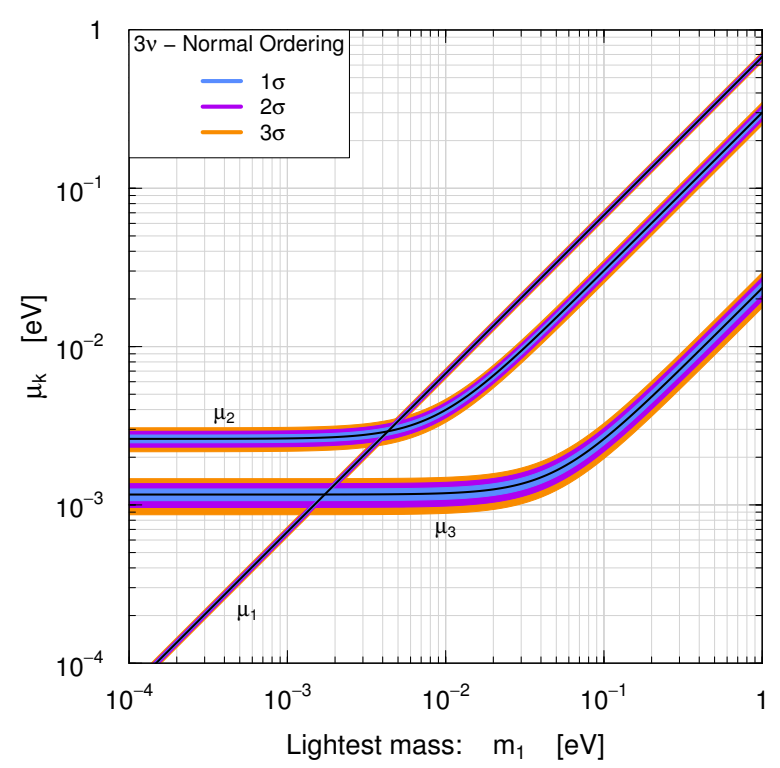

Figure 1. Best-fit values (b.f.) and $1 \sigma, 2 \sigma$ and $3 \sigma$ allowed intervals of the three partial mass contributions to $\left|m_{\beta \beta}\right|$ in eq. (2.1) as functions of the lightest mass $m_{1}$ in the case of $3 \nu$ mixing with Normal Ordering.

From figure 1 one can see that for $m_{1} \lesssim 2 \times 10^{-3} \mathrm{eV}$ the contribution of $\mu_{2}$ is dominant and cannot be canceled by the smaller contributions of $\mu_{1}$ and $\mu_{3}$ for any values of the relative phase differences. In the interval $m_{1} \approx(2-7) \times 10^{-3} \mathrm{eV}$ cancellations are possible, mainly between $\mu_{1}$ and $\mu_{2}$ which have similar values, with the smaller contribution of $\mu_{3}$, which is about 2.3 times smaller than $\mu_{2}$. For $m_{1} \gtrsim 7 \times 10^{-3} \mathrm{eV}$ again there cannot be a complete cancellation, because the contribution of $\mu_{1}$ is dominant.

The result for $\left|m_{\beta \beta}\right|$ is shown in figure 3, where we have plotted separately the allowed bands for the four possible cases in which CP is conserved $\left(\alpha_{2}, \alpha_{3}=0, \pi\right)$ and the coefficients of the contributions are real. These are the extreme cases which determine the minimum and maximum values of $\left|m_{\beta \beta}\right|$. The areas between the CP-conserving curves correspond to values of $\left|m_{\beta \beta}\right|$ which are allowed only in the case of CP violation [31, 50-54] (although there is no manifest $\mathrm{CP}$ violation [55]).

Figure 3 shows also the $90 \%$ C.L. upper limit band for $\left|m_{\beta \beta}\right|$ estimated in ref. [22] from the results of the KamLAND-Zen experiment [56] taking into account the uncertainties of the nuclear matrix element calculations. The reliability of this upper limit is supported by the upper limits with the same order of magnitude following from the results of the Heidelberg-Moscow [57], IGEX [58], GERDA [59], NEMO-3 [60], CUORICINO [61], and EXO [62] experiments.

From figure 3 one can see that, in agreement with the discussion above, there can be a complete cancellation of the three partial mass contributions to $\left|m_{\beta \beta}\right|$ for $m_{1}$ in the intervals given in table 1 at different confidence levels.

The exact determination of the region in which there can be a complete cancellation of the partial mass contributions to $\left|m_{\beta \beta}\right|$ in the general case of $N$-neutrino mixing can be 
done in the following relatively simple way. ${ }^{2}$ For each value of the lightest mass $m_{1}$ let us denote by $a$ the index of the largest mass contribution, i.e.

$$
\mu_{a} \geq \mu_{k} \quad \text { for } \quad k \neq a .
$$

Then, we can consider the quantities

$$
m_{\beta \beta}^{( \pm)}=\mu_{a} \pm \sum_{k \neq a} \mu_{k}
$$

The quantity $m_{\beta \beta}^{(+)}$is always positive and represents the most favorable case, in which all the mass contribution add with the same phase, giving the maximum value of $\left|m_{\beta \beta}\right|$ for any value of the unknown phases:

$$
\left|m_{\beta \beta}\right|_{\max }=m_{\beta \beta}^{(+)} .
$$

The quantity $m_{\beta \beta}^{(-)}$represents the extreme case in which the phases of all the other partial mass contributions are equal and opposite to the phase of the largest mass contribution. It is evident that if $m_{\beta \beta}^{(-)}>0$, the value of $m_{\beta \beta}$ gives the minimum possible value of $\left|m_{\beta \beta}\right|$ for any value of the unknown phases, because it corresponds to the maximal cancellation between $\mu_{a}$ and the maximum $\sum_{k \neq a} \mu_{k}$ of the other partial mass contributions. On the other hand, if

$$
m_{\beta \beta}^{(-)} \leq 0,
$$

there is an intermediate value of the phases which gives $\left|m_{\beta \beta}\right|=0$. This can be seen clearly by writing $\left|m_{\beta \beta}\right|$ as

$$
\left|m_{\beta \beta}\right|=\left|\mu_{a}+e^{i \alpha^{\prime}} \mu^{\prime}\right|
$$

with

$$
\mu^{\prime}=\left|\sum_{k \neq a} e^{i \xi_{k}} \mu_{k}\right|,
$$

with an unknown phase $\alpha^{\prime}$ and $N-2$ unknown phases $\xi_{k}$, of which one can be fixed to zero. The only possibility to have $\left|m_{\beta \beta}\right|=0$ can be realized for $\alpha^{\prime}=\pi$ if $\mu^{\prime}=\mu_{a}$. This equality can occur only if

$$
\mu_{\min }^{\prime} \leq \mu_{a} \leq \mu_{\max }^{\prime}
$$

where $\mu_{\min }^{\prime}$ and $\mu_{\max }^{\prime}$ are the minimum and maximum values of $\mu^{\prime}$ for any value of the unknown phases $\xi_{k}$. Since we have always $\mu_{\min }^{\prime} \leq \mu_{a}$ because of eq. (2.12) and

$$
\mu_{\max }^{\prime}=\sum_{k \neq a} \mu_{k}
$$

the inequality in eq. (2.15) is the necessary and sufficient condition for having $\left|m_{\beta \beta}\right|=0$ for some value of the unknown phases. Hence, the minimum value of $\left|m_{\beta \beta}\right|$ is given by

$$
\left|m_{\beta \beta}\right|_{\min }=\max \left[m_{\beta \beta}^{(-)}, 0\right],
$$

\footnotetext{
${ }^{2}$ Other ways are discussed in refs. [30, 63, 64].
} 


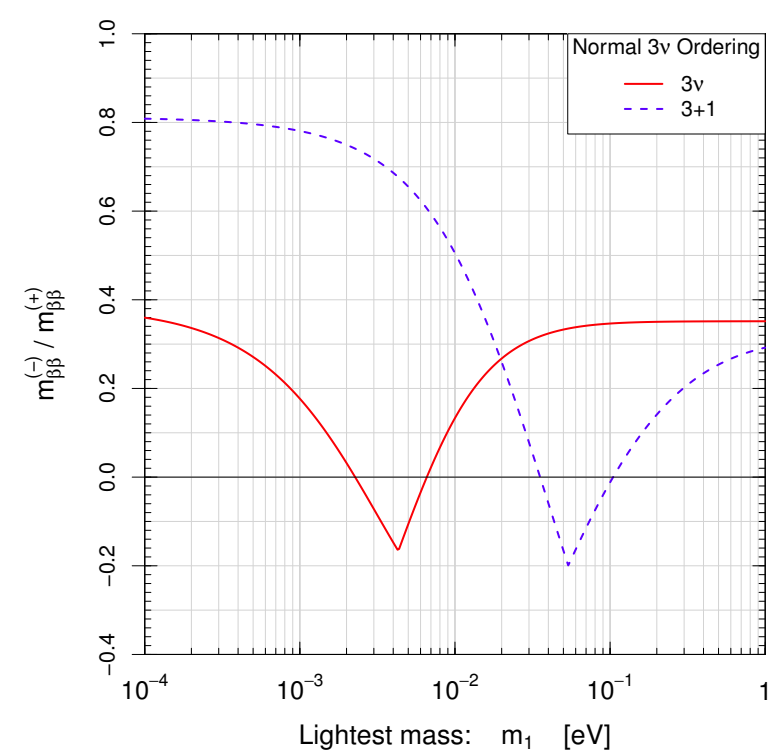

Figure 2. Ratio $m_{\beta \beta}^{(-)} / m_{\beta \beta}^{(+)}$(see eq. (2.13)) as a function of $m_{1}$ for the best-fit values of the partial mass contributions in the case of $3 \nu$ and $3+1$ mixing with Normal Ordering of the three lightest neutrinos.

\begin{tabular}{|ccccc|}
\hline & b.f. & $1 \sigma$ & $2 \sigma$ & $3 \sigma$ \\
\hline$m_{1}\left[10^{-3} \mathrm{eV}\right]$ & $2.3-6.6$ & $1.9-7.2$ & $1.6-8.0$ & $1.3-9.0$ \\
$m_{\beta}\left[10^{-2} \mathrm{eV}\right]$ & $0.9-1.1$ & $0.9-1.2$ & $0.8-1.3$ & $0.8-1.4$ \\
$\Sigma\left[10^{-2} \mathrm{eV}\right]$ & $6.1-6.8$ & $5.9-7.0$ & $5.8-7.2$ & $5.7-7.4$ \\
\hline
\end{tabular}

Table 1. Ranges of $m_{1}, m_{\beta}$ and $\Sigma$ for which there can be a complete cancellation of the three partial mass contributions to $\left|m_{\beta \beta}\right|$ for the best-fit values (b.f.) of the oscillation parameters and at $1 \sigma, 2 \sigma$ and $3 \sigma$ in the case of $3 \nu$ mixing with Normal Ordering.

which can also be written as $[63]^{3}$

$$
\left|m_{\beta \beta}\right|_{\min }=\max \left[2 \mu_{k}-\left|m_{\beta \beta}\right|_{\max }, 0\right] .
$$

Figure 2 shows the value of the ratio $m_{\beta \beta}^{(-)} / m_{\beta \beta}^{(+)}$as a function of $m_{1}$ for the bestfit values of the partial mass contributions, which is sufficient for the determination of the interval of $m_{1}$ for which there can be a complete cancellation of the partial mass contributions. One can see that in the case of $3 \nu$ mixing $m_{\beta \beta}^{(-)}$is negative and it is possible that $\left|m_{\beta \beta}\right|=0$ only in the interval of $m_{1}$ given in table 1 .

Let us now consider the opposite possibility that $\left|m_{\beta \beta}\right|$ is larger than about $0.01 \mathrm{eV}$, which is a value that may be explored experimentally in the near future. From figure 3 one can see that $\left|m_{\beta \beta}\right| \gtrsim 0.01 \mathrm{eV}$ can be realized only for $m_{1} \gtrsim 0.008 \mathrm{eV}$. This range of $m_{1}$ corresponds to almost degenerate $m_{1}$ and $m_{2}$, because $\sqrt{\Delta m_{\text {SOL }}^{2}} \approx 8.7 \times 10^{-3} \mathrm{eV}$. Hence,

\footnotetext{
${ }^{3}$ Equation (2.21) is the generalization to $N$-neutrino mixing of that obtained with a different proof in ref. [63] in the case of $3 \nu$ mixing.
} 


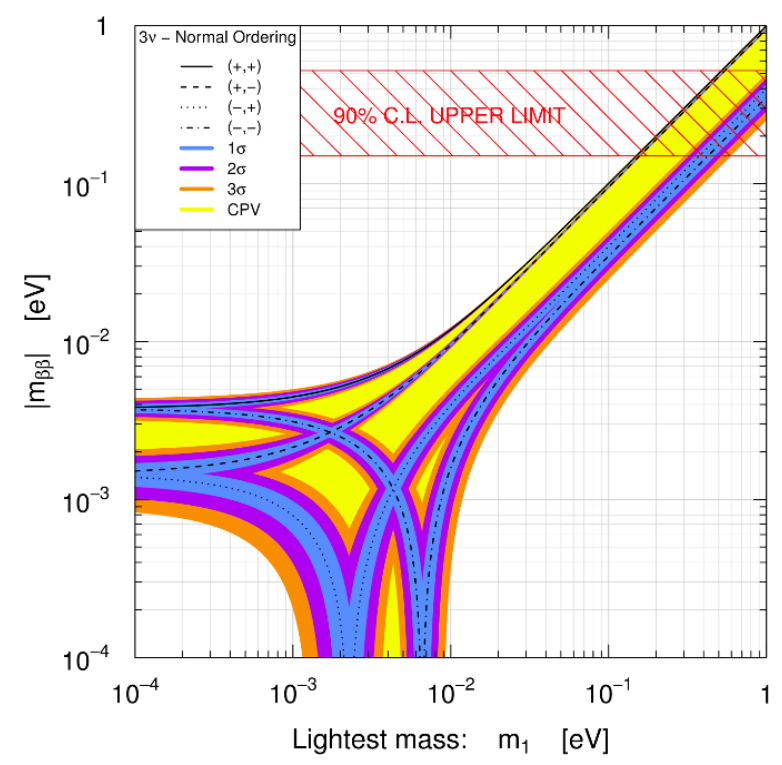

Figure 3. Value of the effective Majorana mass $\left|m_{\beta \beta}\right|$ as a function of the lightest neutrino mass $m_{1}$ in the case of $3 \nu$ mixing with Normal Ordering. The signs in the legend indicate the signs of $e^{i \alpha_{2}}, e^{i \alpha_{3}}= \pm 1$ for the four possible cases in which $\mathrm{CP}$ is conserved. The intermediate yellow region is allowed only in the case of $\mathrm{CP}$ violation. The $90 \%$ upper limit is explained in the main text.

it will be very difficult to measure $\left|m_{\beta \beta}\right|$ if there is a normal hierarchy of neutrino masses $\left(m_{1} \ll m_{2} \ll m_{3}\right)$ for any value of the unknown phases $\alpha_{2}$ and $\alpha_{3}$ in eq. (2.1).

One can also see from figure 3 that $\left|m_{\beta \beta}\right| \gtrsim 0.01 \mathrm{eV}$ is realized independently of the values of the unknown phases $\alpha_{2}$ and $\alpha_{3}$ for $m_{1} \gtrsim 0.04 \mathrm{eV}$, which is close to the region $m_{1} \gtrsim \sqrt{\Delta m_{\mathrm{ATM}}^{2}} \approx 0.05 \mathrm{eV}$ in which all the three neutrino masses are quasidegenerate.

Figure 3 gives a clear view of the possible values of $\left|m_{\beta \beta}\right|$ depending on the scale of the lightest mass $m_{1}$, but it is of little practical usefulness, because it will be very difficult to measure directly the value of $m_{1}$. In practice, the investigation of the absolute values of neutrino masses is performed through the measurements of the effective electron neutrino mass

$$
m_{\beta}=\sqrt{\left|U_{e 1}\right|^{2} m_{1}^{2}+\left|U_{e 2}\right|^{2} m_{2}^{2}+\left|U_{e 3}\right|^{2} m_{3}^{2}}
$$

in $\beta$-decay experiments $[23,24]$ and through the measurement of the sum of the neutrino masses

$$
\Sigma=m_{1}+m_{2}+m_{3}
$$

in cosmological experiments (see, for example, ref. [65]). Hence, it is useful to calculate the allowed regions in the $m_{\beta}-\left|m_{\beta \beta}\right|$ and $\Sigma-\left|m_{\beta \beta}\right|$ planes [66-69], which are shown in figures 4 and 5 . In this case, the confidence intervals are calculated using the $\chi^{2}$ function in eq. (2.11) with two degrees of freedom. We have plotted separately the allowed bands for the four possible cases in which CP is conserved $\left(\alpha_{2}, \alpha_{3}=0, \pi\right)$, in order to show the regions in which $\mathrm{CP}$ is violated. Potentially the possibility of measuring values of $\left|m_{\beta \beta}\right|$ and $m_{\beta}$ or $\Sigma$ in these regions is very exciting for the discovery of CP violation generated by 


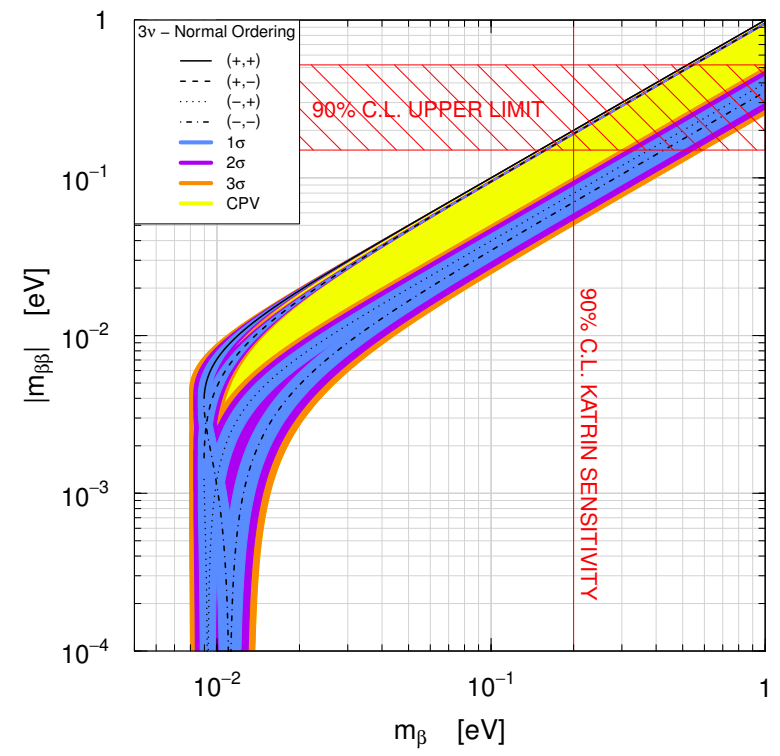

Figure 4. Value of the effective Majorana mass $\left|m_{\beta \beta}\right|$ as a function of effective electron neutrino mass $m_{\beta}$ in eq. (2.22) in the case of $3 \nu$ mixing with Normal Ordering. The legend is explained in the caption of figure 3 . The limits are explained in the main text.

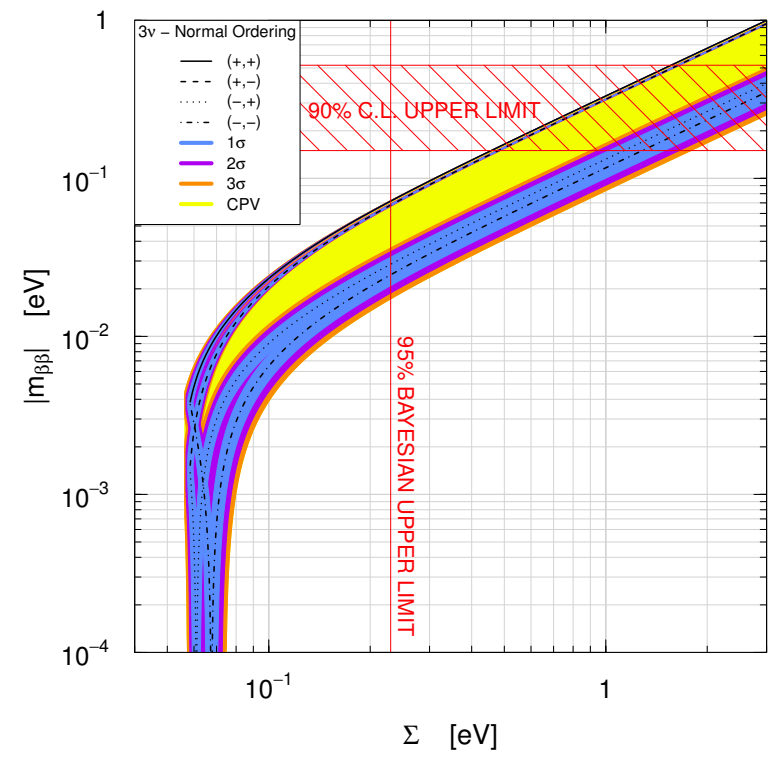

Figure 5. Value of the effective Majorana mass $\left|m_{\beta \beta}\right|$ as a function of sum of the neutrino masses $\Sigma$ in eq. (2.23) in the case of $3 \nu$ mixing with Normal Ordering. The legend is explained in the caption of figure 3 . The limits are explained in the main text. 
the Majorana phases, ${ }^{4}$ but in practice such measurement is very difficult because it would require a precision which seems to be beyond what can be currently envisioned [71-74], especially taking into account the current uncertainty of the calculation of the nuclear matrix element in neutrinoless double- $\beta$ decay (see refs. [22, 75-77]).

Figures 4 and 5 show the same $90 \%$ C.L. upper limit band for $\left|m_{\beta \beta}\right|$ as in figure 3. In addition, figure 4 shows $^{5}$ the $90 \%$ C.L. sensitivity on $m_{\beta}$ of the KATRIN experiment [78], which is scheduled to start data taking in 2016, and figure 5 shows the $95 \%$ bayesian upper limit on $\Sigma$ obtained by the Planck collaboration [65].

The intervals of $m_{\beta}$ and $\Sigma$ for which there can be a complete cancellation of the three partial mass contributions to $\left|m_{\beta \beta}\right|$ are given in table 1. On the other hand, from figures 4 and 5 one can see that $\left|m_{\beta \beta}\right| \gtrsim 0.01 \mathrm{eV}$ for any value of the unknown phases $\alpha_{2}$ and $\alpha_{3}$ for $m_{\beta} \gtrsim 0.05 \mathrm{eV}$ and $\Sigma \gtrsim 0.15 \mathrm{eV}$. The $3 \sigma$ lower bounds for $m_{\beta}$ and $\Sigma$ are, respectively, $0.8 \times 10^{-2} \mathrm{eV}$ and $5.6 \times 10^{-2} \mathrm{eV}$.

\section{$2.2 \quad$ Inverted ordering}

In the case of Inverted Ordering (IO), the expressions of the neutrino masses in terms of the lightest neutrino mass $m_{\min }$ are:

$$
\begin{aligned}
& m_{3}=m_{\mathrm{min}}, \\
& m_{1}=\sqrt{m_{\mathrm{min}}^{2}+\Delta m_{\mathrm{ATM}}^{2}-\Delta m_{\mathrm{SOL}}^{2} / 2}, \\
& m_{2}=\sqrt{m_{\mathrm{min}}^{2}+\Delta m_{\mathrm{ATM}}^{2}+\Delta m_{\mathrm{SOL}}^{2} / 2} .
\end{aligned}
$$

Figure 6 shows the best-fit values and the $1 \sigma, 2 \sigma$ and $3 \sigma$ allowed intervals of the three partial mass contributions to $\left|m_{\beta \beta}\right|$ in eq. (2.1) as functions of the lightest mass $m_{3}$. One can see that $\mu_{1}$ is always dominant, because $\vartheta_{12}$ is smaller than $\pi / 4$ and $\left|U_{e 1}\right|>\left|U_{e 2}\right|>\left|U_{e 3}\right|$. Therefore, in the case of Inverted Ordering there cannot be a complete cancellation of the three mass contributions to $\left|m_{\beta \beta}\right|$ (figure 8 shows that $m_{\beta \beta}^{(-)}$is always positive) and we obtain from figure 7 the lower bounds

$$
\left|m_{\beta \beta}\right|>1.6(1 \sigma), 1.5(2 \sigma), 1.3(3 \sigma) \times 10^{-2} \mathrm{eV} .
$$

In the case of an Inverted Hierarchy $\left(m_{3} \ll m_{1}<m_{2}\right)$ we also have the upper bounds

$$
\left|m_{\beta \beta}\right|<4.8(1 \sigma), 4.9(2 \sigma), 4.9(3 \sigma) \times 10^{-2} \mathrm{eV} .
$$

The next generations of neutrinoless double-beta decay experiments (see refs. [36-41]) will try to explore the range of $\left|m_{\beta \beta}\right|$ between the limits in eqs. (2.27) and (2.28), testing the Majorana nature of neutrinos in the case of an Inverted Hierarchy.

\footnotetext{
${ }^{4}$ The phases $\alpha_{2}$ and $\alpha_{3}$ in eq. (2.1) depend on the values of one Dirac phase and two Majorana phases in the neutrino mixing matrix (see ref. [70]). The Dirac phase can be measured in neutrino oscillation experiments and there is some indication on its value [3-5]. On the other hand, the values of the two Majorana phases can be measured only in lepton-number violating processes such as neutrinoless double- $\beta$ decay. In the future, if the Dirac phase will be measured, CP violation in neutrinoless double- $\beta$ decay may provide information on the Majorana phases.

${ }^{5}$ The most stringent current upper limits on $m_{\beta}$ obtained in the Mainz $\left(m_{\beta}<2.3 \mathrm{eV}\right.$ at $95 \%$ C.L.) [23] and Troitsk $\left(m_{\beta}<2.1 \mathrm{eV} 95 \%\right.$ C.L.) [24] experiments are out of the scale in figure 4.
} 


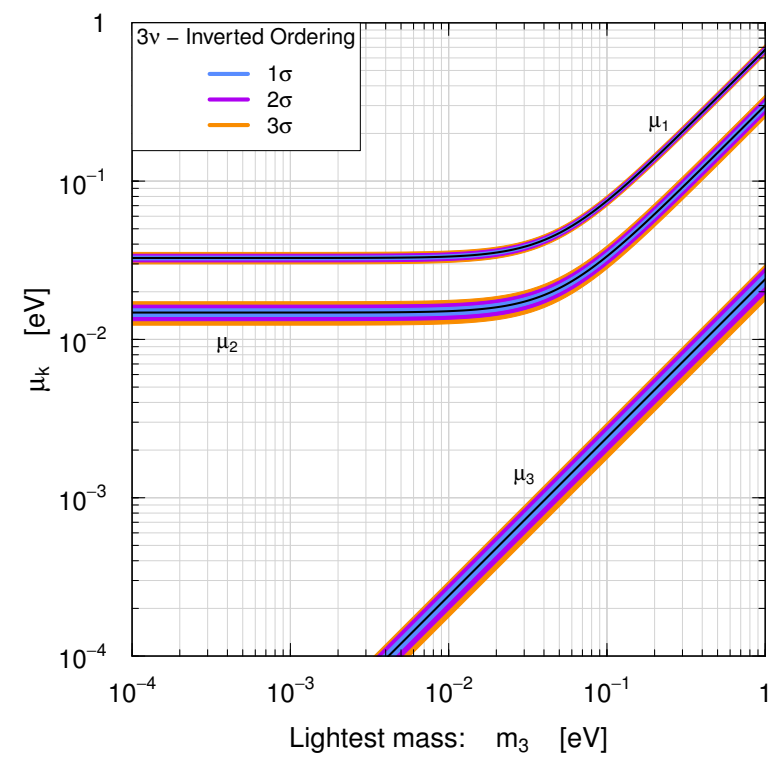

Figure 6. Best-fit values (b.f.) and $1 \sigma, 2 \sigma$ and $3 \sigma$ allowed intervals of the three partial mass contributions to $\left|m_{\beta \beta}\right|$ in eq. (2.1) as functions of the lightest mass $m_{3}$ in the case of $3 \nu$ mixing with Inverted Ordering.

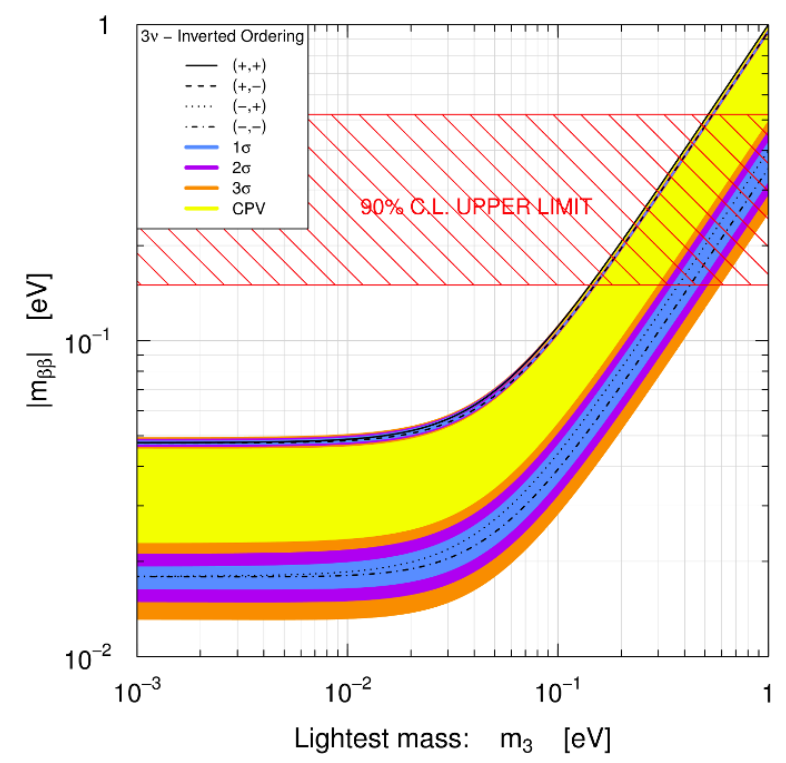

Figure 7. Value of the effective Majorana mass $\left|m_{\beta \beta}\right|$ as a function of lightest neutrino mass in the three neutrino case for the Inverted Ordering. The legend is explained in the caption of figure 3. The $90 \%$ upper limit is explained in section 2.1. 


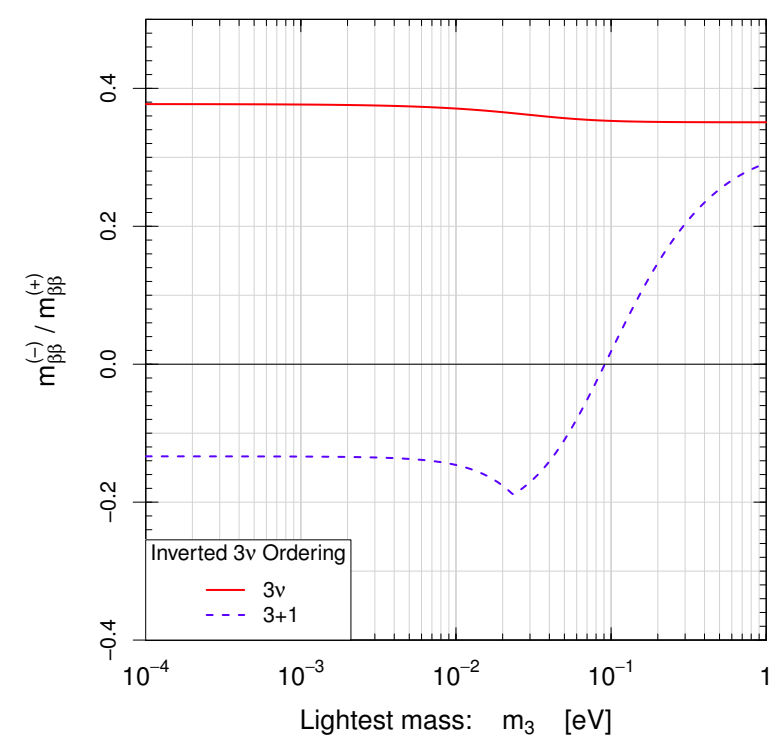

Figure 8. Ratio $m_{\beta \beta}^{(-)} / m_{\beta \beta}^{(+)}$(see eq. (2.13)) as a function of $m_{3}$ for the best-fit values of the partial mass contributions in the case of $3 \nu$ and $3+1$ mixing with Inverted Ordering of the three lightest neutrinos.

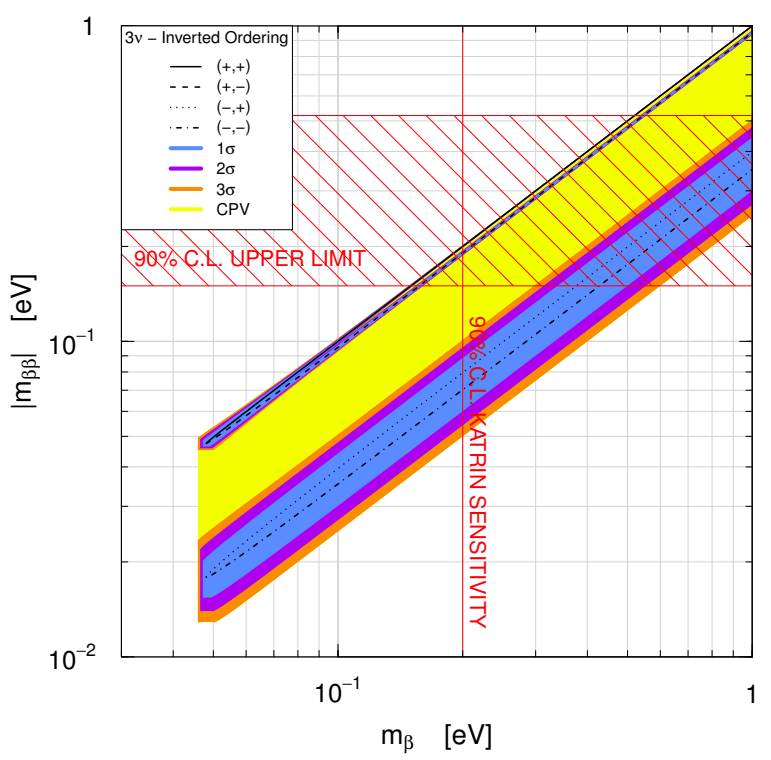

Figure 9. Value of the effective Majorana mass $\left|m_{\beta \beta}\right|$ as a function of effective electron neutrino mass $m_{\beta}$ in eq. (2.22) in the case of $3 \nu$ mixing with Inverted Ordering. The legend is explained in the caption of figure 3. The limits are explained in in section 2.1 .

Figures 9 and 10 show the correlation between $\left|m_{\beta \beta}\right|$ and the measurable quantities $m_{\beta}$ and $\Sigma$ in the Inverted Ordering. Since in this case both $m_{\beta}$ and $\Sigma$ have relatively large lower bounds $\left(4.6 \times 10^{-2} \mathrm{eV}\right.$ and $9.4 \times 10^{-2} \mathrm{eV}$, respectively, at $\left.3 \sigma\right)$ there is a concrete possibility that near-future experiments will determine an allowed region in these plots if in nature there are only three neutrinos with Inverted Ordering. 


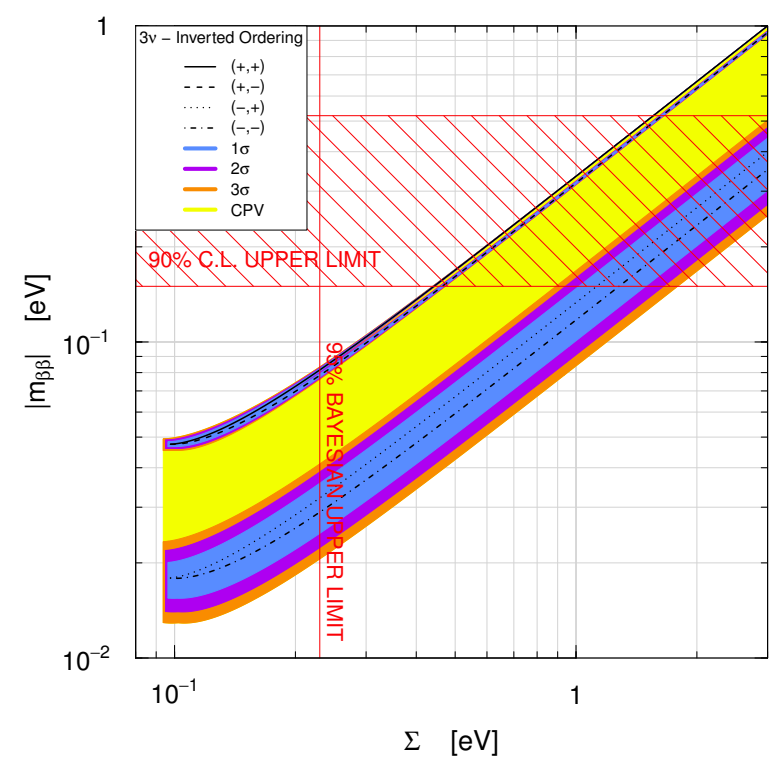

Figure 10. Value of the effective Majorana mass $\left|m_{\beta \beta}\right|$ as a function of sum of the neutrino masses $\Sigma$ in eq. (2.23) in the case of $3 \nu$ mixing with Inverted Ordering. The legend is explained in the caption of figure 3. The limits are explained in in section 2.1 .

\section{$3 \quad 3+1$ mixing}

In this section we consider the case of $3+1$ mixing in which there is a new massive neutrino $\nu_{4}$ at the $\mathrm{eV}$ scale which is mainly sterile. As explained in section $1,3+1$ mixing is motivated $[18,19]$ by the explanation of the reactor, Gallium and LSND anomalies, which requires the existence of a new squared-mass difference $\Delta m_{\mathrm{SBL}}^{2} \sim 1 \mathrm{eV}^{2}$. In this case, the effective Majorana mass in neutrinoless double-beta decay is given by

$$
\left|m_{\beta \beta}\right|=\left|\mu_{1}+\mu_{2} e^{i \alpha_{2}}+\mu_{3} e^{i \alpha_{3}}+\mu_{4} e^{i \alpha_{4}}\right|,
$$

with the partial mass contributions given by eq. (2.2). The contribution of $\nu_{4}$ enters with a totally unknown new phase $\alpha_{4}$ that must be varied from 0 to $2 \pi$ as $\alpha_{2}$ and $\alpha_{3}$ in order to calculate the predictions of the value of $\left|m_{\beta \beta}\right|$.

The absolute values of the relevant first row of the $4 \times 4$ mixing matrix $U$ is given by the simple extension of the standard parameterization:

$$
\begin{aligned}
& \left|U_{e 1}\right|=\cos \vartheta_{14} \cos \vartheta_{13} \cos \vartheta_{12}, \\
& \left|U_{e 2}\right|=\cos \vartheta_{14} \cos \vartheta_{13} \sin \vartheta_{12}, \\
& \left|U_{e 3}\right|=\cos \vartheta_{14} \sin \vartheta_{13}, \\
& \left|U_{e 4}\right|=\sin \vartheta_{14} .
\end{aligned}
$$

Since in the case of $3+1$ neutrino mixing, as well as in any extension of the standard $3 \nu$ mixing, the ordering of the three standard massive neutrinos is not known, in the following two subsections we consider separately the two cases of Normal and Inverted Ordering of $\nu_{1}, \nu_{2}, \nu_{3}$. The values of their masses as functions of the lightest mass $m_{\min }$ are given by 


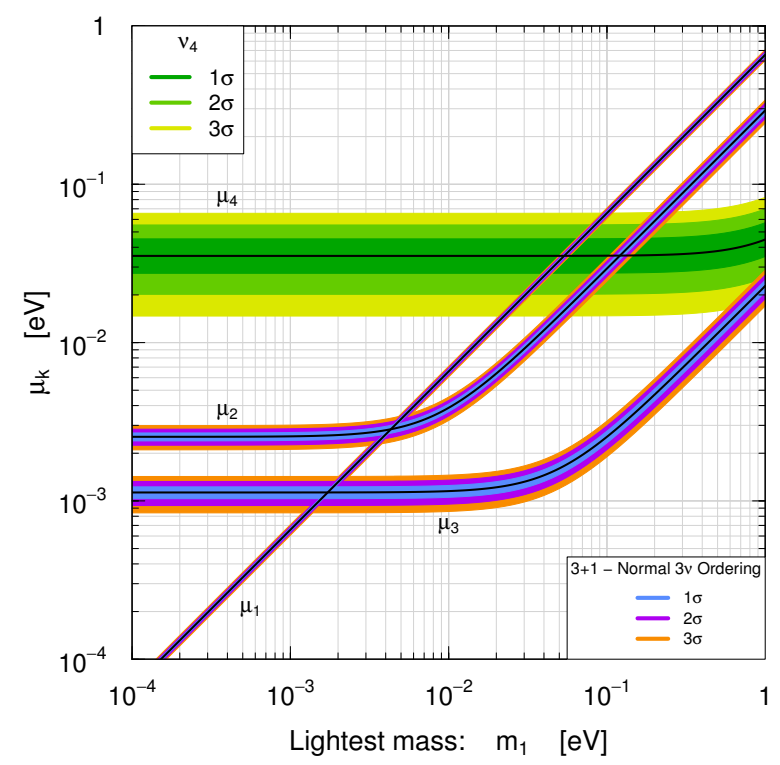

Figure 11. Best-fit values (b.f.) and $1 \sigma, 2 \sigma$ and $3 \sigma$ allowed intervals of the four partial mass contributions to $\left|m_{\beta \beta}\right|$ in eq. (3.1) as functions of the lightest mass $m_{1}$ in the case of $3+1$ mixing with Normal Ordering of the three lightest neutrinos.

eqs. (2.8) $-(2.10)$ in the Normal Ordering and by eqs. (2.24)-(2.26) in the Inverted Ordering. However, in both cases we have

$$
m_{4} \simeq \sqrt{m_{\min }^{2}+\Delta m_{\mathrm{SBL}}^{2}}
$$

neglecting the contributions of $\Delta m_{\mathrm{SOL}}^{2}$ and $\Delta m_{\mathrm{ATM}}^{2}$, which are much smaller than $\Delta m_{\mathrm{SBL}}^{2}$. We calculated the confidence intervals using the $\chi^{2}$ function

$$
\chi_{3+1}^{2}=\chi_{3 \nu}^{2}+\chi^{2}\left(\Delta m_{\mathrm{SBL}}^{2}, \sin ^{2} \vartheta_{14}\right),
$$

with $\chi_{3 \nu}^{2}$ defined in eq. $(2.11)$ and $\chi^{2}\left(\Delta m_{\mathrm{SBL}}^{2}, \sin ^{2} \vartheta_{14}\right)$ obtained from an update [34, 35] of the global fit of short-baseline neutrino oscillation data presented in ref. [19].

After eq. (2.11) we noted that in the case of $3 \nu$ mixing our statistical method for the calculation of the uncertainty of $\left|m_{\beta \beta}\right|$ and the usual method based on the propagation of errors lead to similar results, because the $\chi^{2}$ 's of the relevant $3 \nu$ mixing parameters are very well approximated by quadratic functions. On the other hand, the usual propagation of errors is inaccurate in the case of $3+1$ mixing, because the marginal $\chi^{2}$ 's of $\Delta m_{\mathrm{SBL}}^{2}$ and $\sin ^{2} \vartheta_{14}$ are not quadratic. Moreover, there are significant correlations between $\Delta m_{\mathrm{SBL}}^{2}$ and $\sin ^{2} \vartheta_{14}$ (see figure 3 of ref. [19]) which are taken into account in $\chi^{2}\left(\Delta m_{\mathrm{SBL}}^{2}, \sin ^{2} \vartheta_{14}\right)$.

\subsection{Normal ordering}

Figure 11 shows a comparison of the best-fit value and the $1 \sigma, 2 \sigma$ and $3 \sigma$ allowed intervals of the partial contribution $\mu_{4}$ as a function of the lightest mass $m_{1}$ with those of $\mu_{1}, \mu_{2}$, $\mu_{3}$, which are slightly different from those in figure 1 because of the contribution of $\vartheta_{14}$ in eqs. (3.2)-(3.4). One can see that in the $3+1$ case it is not possible to get a total 


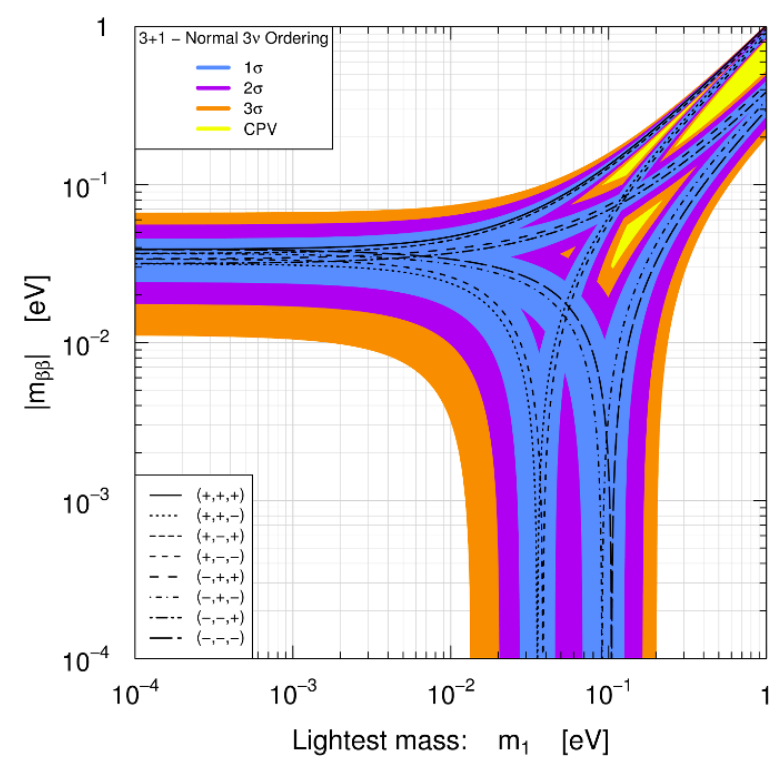

Figure 12. Value of the effective Majorana mass $\left|m_{\beta \beta}\right|$ as a function of the lightest neutrino mass $m_{1}$ in the case of $3+1$ mixing with Normal Ordering of the three lightest neutrinos. The signs in the legend indicate the signs of $e^{i \alpha_{2}}, e^{i \alpha_{3}}, e^{i \alpha_{4}}= \pm 1$ for the four possible cases in which $\mathrm{CP}$ is conserved. The intermediate yellow region is allowed only in the case of $\mathrm{CP}$ violation.

\begin{tabular}{|ccccc|}
\hline & b.f. & $1 \sigma$ & $2 \sigma$ & $3 \sigma$ \\
\hline$m_{1}\left[10^{-2} \mathrm{eV}\right]$ & $3.5-10.5$ & $2.8-12.5$ & $2.0-16.3$ & $1.3-20.0$ \\
$m_{\beta}\left[10^{-2} \mathrm{eV}\right]$ & $3.6-10.5$ & $2.6-14.3$ & $1.9-17.8$ & $1.5-22.7$ \\
$\Sigma\left[10^{-2} \mathrm{eV}\right]$ & $13.3-32.6$ & $10.4-43.8$ & $8.7-53.9$ & $7.6-68.5$ \\
\hline
\end{tabular}

Table 2. Ranges of $m_{1}, m_{\beta}$ and $\Sigma$ for which there can be a complete cancellation of the four partial mass contributions to $\left|m_{\beta \beta}\right|$ for the best-fit values (b.f.) of the oscillation parameters and at $1 \sigma, 2 \sigma$ and $3 \sigma$ in the case of $3+1$ mixing with Normal Ordering of the three lightest neutrinos.

cancellation of $\left|m_{\beta \beta}\right|$ in the interval $m_{1} \approx(2-7) \times 10^{-3} \mathrm{eV}$ as in the case of $3 \nu$ mixing (see table 1), because in this interval of $m_{1}$ the contribution of $\mu_{4}$ is dominant. However, there is a range of higher values of $m_{1}$ between about 0.02 and $0.2 \mathrm{eV}$ in which $\mu_{4}$ and $\mu_{1}$ have similar values, leading to a possible total cancellation. For $m_{1} \gtrsim 0.2 \mathrm{eV}$ a total cancellation is again not possible because the contribution of $\mu_{1}$ is dominant. This behavior is confirmed by figure 2 , where one can see that the value of $m_{\beta \beta}^{(-)}$(see eq. (2.13)) corresponding to the best-fit values of the partial mass contributions is negative for $0.035 \lesssim m_{1} \lesssim 0.1 \mathrm{eV}$.

Figure 12 shows the allowed values of $\left|m_{\beta \beta}\right|$ as a function of $m_{1}$ at different confidence levels. The corresponding intervals of $m_{1}$ for which there can be a total cancellation of $\left|m_{\beta \beta}\right|$ are given in table 2 .

In figure 12 we have plotted separately the allowed bands for the eight possible cases in which CP is conserved $\left(\alpha_{2}, \alpha_{3}, \alpha_{4}=0, \pi\right)$, that are the extreme cases which determine the minimum and maximum values of $\left|m_{\beta \beta}\right|$. The areas between the CP-conserving allowed bands correspond to values of $\left|m_{\beta \beta}\right|$ which are allowed only in the case of CP violation. 
Unfortunately, these areas are visibly smaller than those in figure 3 in the case of $3 \nu$ mixing. This is due to the relatively large uncertainty of $\mu_{4}$, which can be seen clearly in figure 11 . This uncertainty broadens the allowed bands corresponding to the CP-conserving cases, leaving little intermediate space. In any case, even if the uncertainty of $\mu_{4}$ will be reduced in the future, there cannot be a region which is allowed only in the case of CP-violation for $m_{1} \lesssim 10^{-2} \mathrm{eV}$, where $\mu_{4}$ is dominant and the CP-violating phases are irrelevant. In fact, all the best-fit CP-conserving curves have approximately the same value for $m_{1} \lesssim 10^{-2} \mathrm{eV}$.

As in the case of $3 \nu$ mixing, the plot in figure 12 of $\left|m_{\beta \beta}\right|$ as a function of the lightest mass $m_{1}$ is useful because it gives a clear view of the different possibilities for the value of $\left|m_{\beta \beta}\right|$, but in practice it will be very difficult to determine experimentally an allowed region in this plot because of the difficulty of measuring the value of the lightest mass. Therefore, we calculated also the allowed regions in the $m_{\beta}-\left|m_{\beta \beta}\right|$ and $\Sigma-\left|m_{\beta \beta}\right|$ planes shown in figures 13 and 14 , with the quantities $m_{\beta}$ and $\Sigma$ defined in eqs. (2.22) and (2.23) as in the case of $3 \nu$ mixing in terms of the three standard neutrino masses only. The reason of this choice is that $m_{\beta}$ and $\Sigma$ are measurable quantities also in the $3+1$ scheme. Indeed, considering $\beta$ decay, $m_{\beta}$ quantifies approximately the deviation of the end-point of the electron spectrum due to neutrino masses smaller than the experimental energy resolution [79-83], whereas the effect of the larger mass $m_{4}$ is a kink of the Kurie function (see ref. [70]). In cosmology, the effects of the larger mass $m_{4}$ can be disentangled from those of the smaller masses, because $\nu_{4}$ becomes non-relativistic shortly after matter-radiation equality, much earlier than $\nu_{1}, \nu_{2}, \nu_{3}$. Moreover, it is possible that the contribution of $m_{4}$ to the energy density of the Universe is suppressed, for example by a large lepton asymmetry [84-88], or an enhanced background potential due to new interactions in the sterile sector [89-95], or a larger cosmic expansion rate at the time of sterile neutrino production [96], or MeV dark matter annihilation [97].

From figures 13 and 14 one can see that the intervals of $m_{\beta}$ and $\Sigma$ for which there can be a complete cancellation of the three partial mass contributions to $\left|m_{\beta \beta}\right|$ (given in table 2) are much larger than those in figures 4 and 5 for the standard $3 \nu$ mixing case, and $\left|m_{\beta \beta}\right| \gtrsim 0.01 \mathrm{eV}$ for any value of the unknown phases $\alpha_{2}, \alpha_{3}, \alpha_{4}$ only for the relatively large values $m_{\beta} \gtrsim 0.25 \mathrm{eV}$ and $\Sigma \gtrsim 0.8 \mathrm{eV}$.

It is useful to compare the allowed regions $m_{\beta}-\left|m_{\beta \beta}\right|$ and $\Sigma-\left|m_{\beta \beta}\right|$ planes obtained in the cases of $3 \nu$ and $3+1$ mixing with Normal Ordering of the three lightest neutrinos. Figures 15 and 16 show this comparison for the $3 \sigma$ allowed regions. One can see that, if the Normal Ordering will be established by oscillation experiments (see refs. [1, 2]), with measurements of $m_{\beta}$ and $\left|m_{\beta \beta}\right|$ and/or $\Sigma$ and $\left|m_{\beta \beta}\right|$ it may be possible to distinguish $3 \nu$ mixing and $3+1$ mixing if the measured values select a region which is allowed only in one of the two cases. It is interesting that there are two regions allowed only to $3+1$ mixing: one with $\left|m_{\beta \beta}\right|$ smaller than that in the case of $3 \nu$ mixing and one with $\left|m_{\beta \beta}\right|$ larger than that in the case of $3 \nu$ mixing. At least a part of the second region is accessible to the next generation of neutrinoless double-beta decay experiments (see refs. [36-41]). The only $\beta$ decay experiment under preparation with the aim of exploring the sub-eV region of $m_{\beta}$ is KATRIN [98], which will have a sensitivity of about $0.2 \mathrm{eV}$ that is not sufficient to explore the upper part of the region in figure 15 allowed only in the case of $3+1$ mixing. On the 


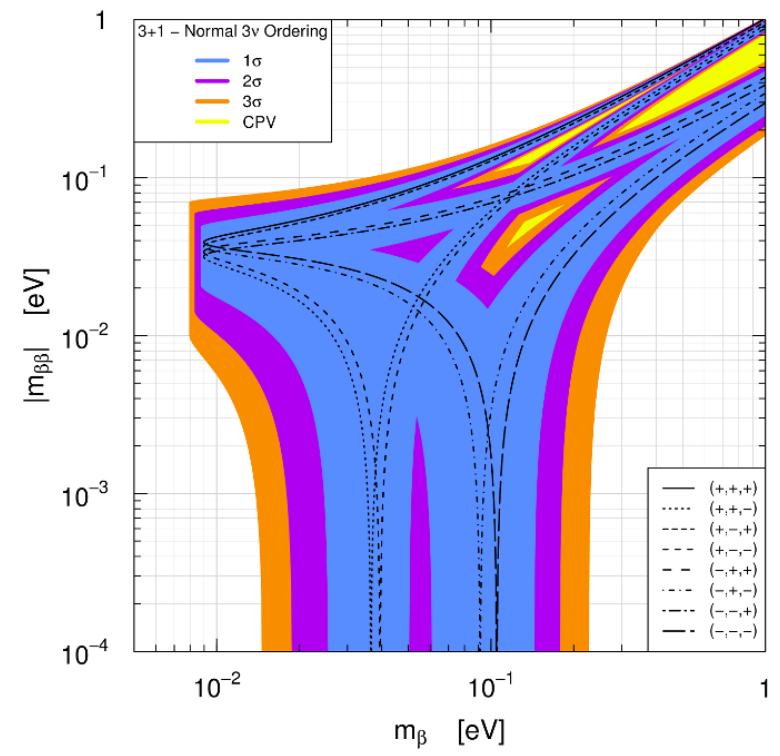

Figure 13. Value of the effective Majorana mass $\left|m_{\beta \beta}\right|$ as a function of effective electron neutrino mass $m_{\beta}$ in eq. (2.22) in the case of $3+1$ mixing with Normal Ordering of the three lightest neutrinos. The legend is explained in the caption of figure 12 .

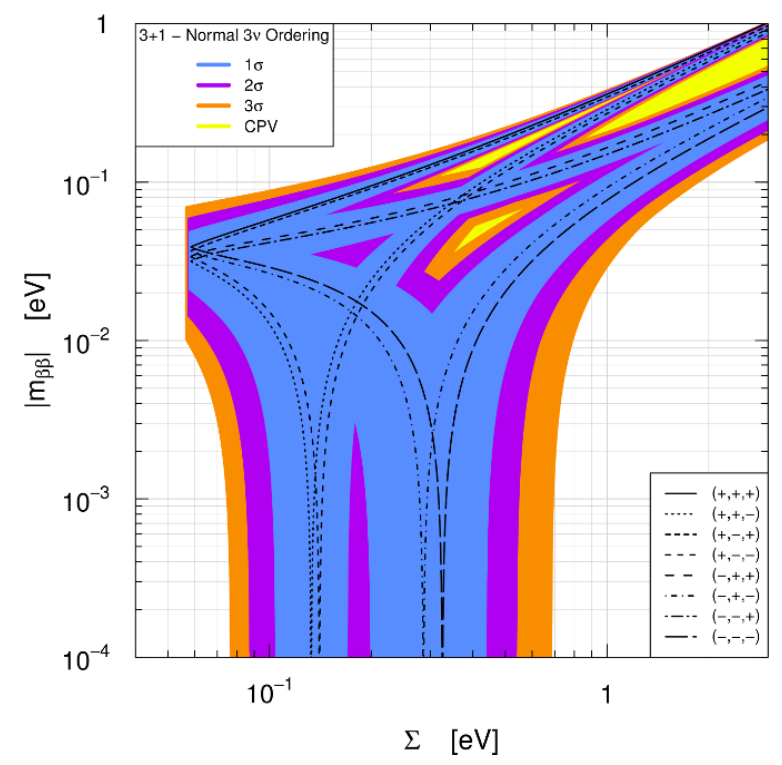

Figure 14. Value of the effective Majorana mass $\left|m_{\beta \beta}\right|$ as a function of sum of the three lightest neutrino masses $\Sigma$ in eq. (2.23) in the case of $3+1$ mixing with Normal Ordering of the three lightest neutrinos. The legend is explained in the caption of figure 12 . 


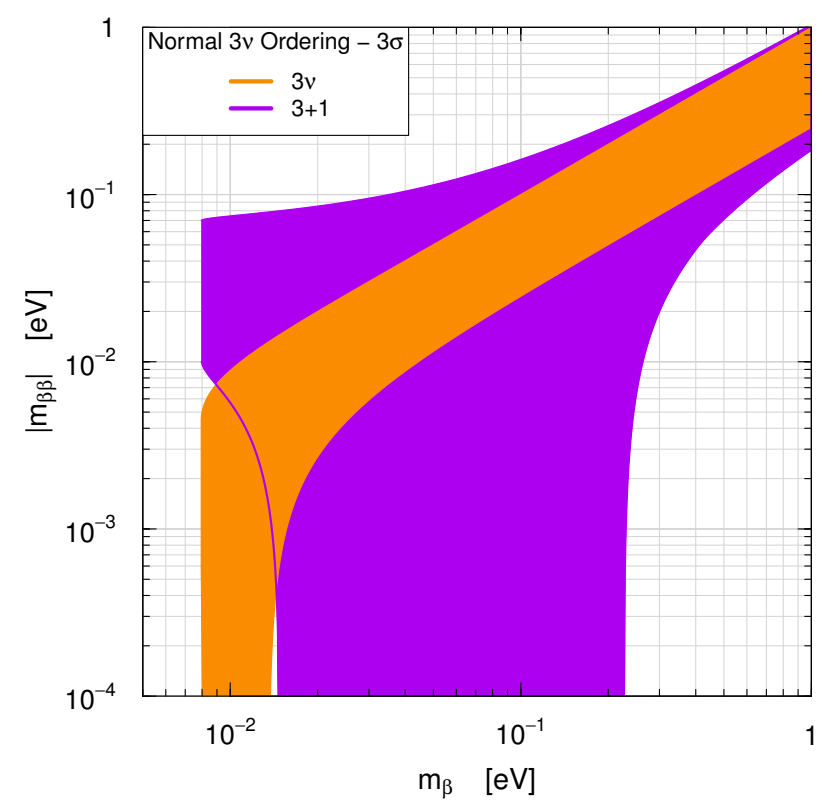

Figure 15. Comparison of the $3 \sigma$ allowed regions in the $m_{\beta}-\left|m_{\beta \beta}\right|$ plane in the cases of $3 \nu$ and $3+1$ mixing with Normal Ordering of the three lightest neutrinos.

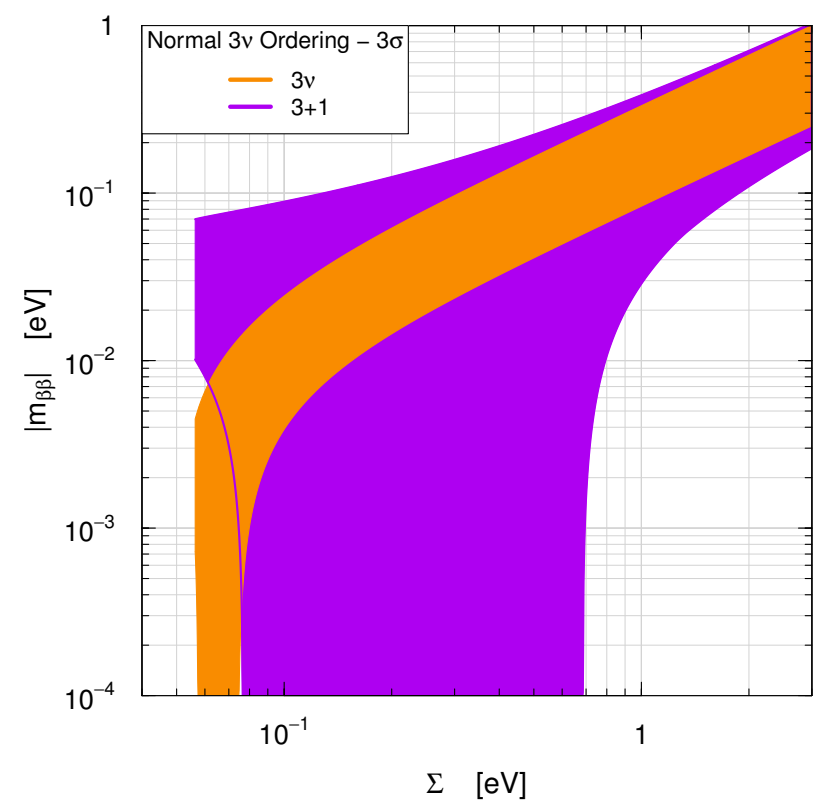

Figure 16. Comparison of the $3 \sigma$ allowed regions in the $\Sigma-\left|m_{\beta \beta}\right|$ plane in the cases of $3 \nu$ and $3+1$ mixing with Normal Ordering of the three lightest neutrinos. 


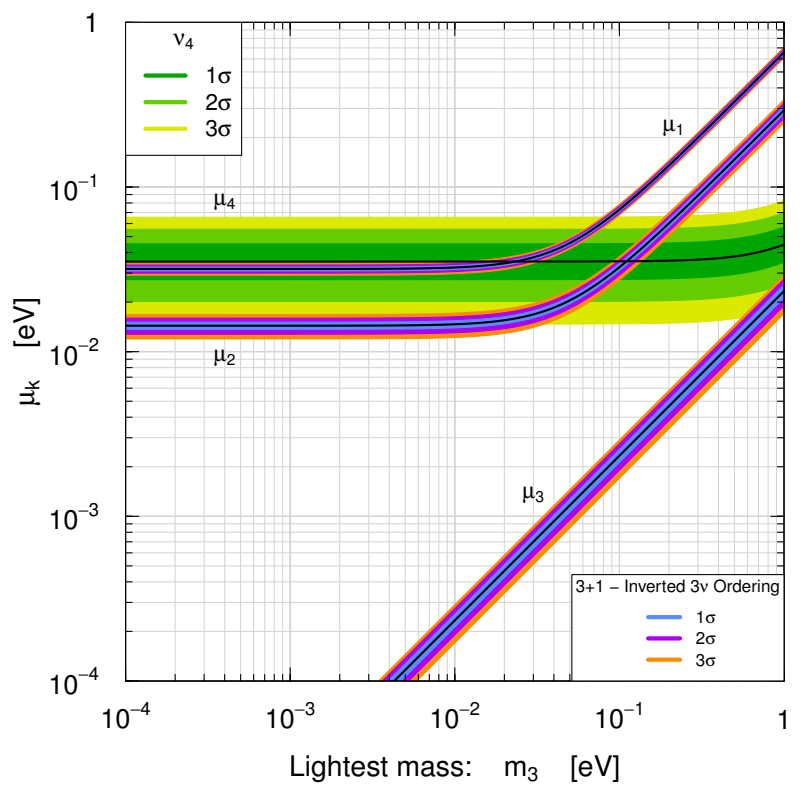

Figure 17. Best-fit values (b.f.) and $1 \sigma, 2 \sigma$ and $3 \sigma$ allowed intervals of the four partial mass contributions to $\left|m_{\beta \beta}\right|$ in eq. (3.1) as functions of the lightest mass $m_{3}$ in the case of $3+1$ mixing with Inverted Ordering of the three lightest neutrinos.

other hand, cosmological observation may be able to measure the sum of the three light neutrino masses down to the lower limit of about $5.6 \times 10^{-2} \mathrm{eV}[99]$.

\subsection{Inverted ordering}

The best-fit value and the $1 \sigma, 2 \sigma$ and $3 \sigma$ allowed intervals of the partial mass contributions to $\left|m_{\beta \beta}\right|$ in the case of $3+1$ mixing with Inverted Ordering of the three lightest neutrinos are shown in figure 17. One can see that there can be a total cancellation between the partial contribution $\mu_{4}$ and the dominant $\mu_{1}$ in the case of $3 \nu$ mixing (see figure 6 ) for $m_{3} \lesssim 0.1 \mathrm{eV}$. Indeed, figure 8 shows that the value of $m_{\beta \beta}^{(-)}$(see eq. (2.13)) corresponding to the best-fit values of the partial mass contributions is negative for $m_{3} \lesssim 0.09 \mathrm{eV}$.

Figure 18 depicts the allowed regions in in the $m_{3}-\left|m_{\beta \beta}\right|$ plane. Comparing figure 18 with figure 7 one can see that the predictions for $\left|m_{\beta \beta}\right|$ are completely different in the $3 \nu$ and $3+1$ cases if there is an Inverted Ordering of the three lightest neutrinos, in agreement with the discussions in refs. [13, 25-33]. The ranges of values of $m_{3}$ for which there can be a complete cancellation of $\left|m_{\beta \beta}\right|$ are given in table 3 .

Figures 19 and 20 show the allowed regions in the $m_{\beta}-\left|m_{\beta \beta}\right|$ and $\Sigma-\left|m_{\beta \beta}\right|$ planes. Figures 21 and 22 show the comparison of the $3 \sigma$ allowed regions in the same planes in the cases of $3 \nu$ and $3+1$ mixing with Inverted Ordering of the three lightest neutrinos. If the Inverted Ordering will be established by oscillation experiments (see refs. [1, 2]), it will be possible to exclude $3 \nu$ mixing in favor of $3+1$ by restricting $m_{\beta}$ and $\left|m_{\beta \beta}\right|$ or $\Sigma$ and $\left|m_{\beta \beta}\right|$ in the corresponding large region at small $\left|m_{\beta \beta}\right|$ allowed only in the $3+1$ case. 


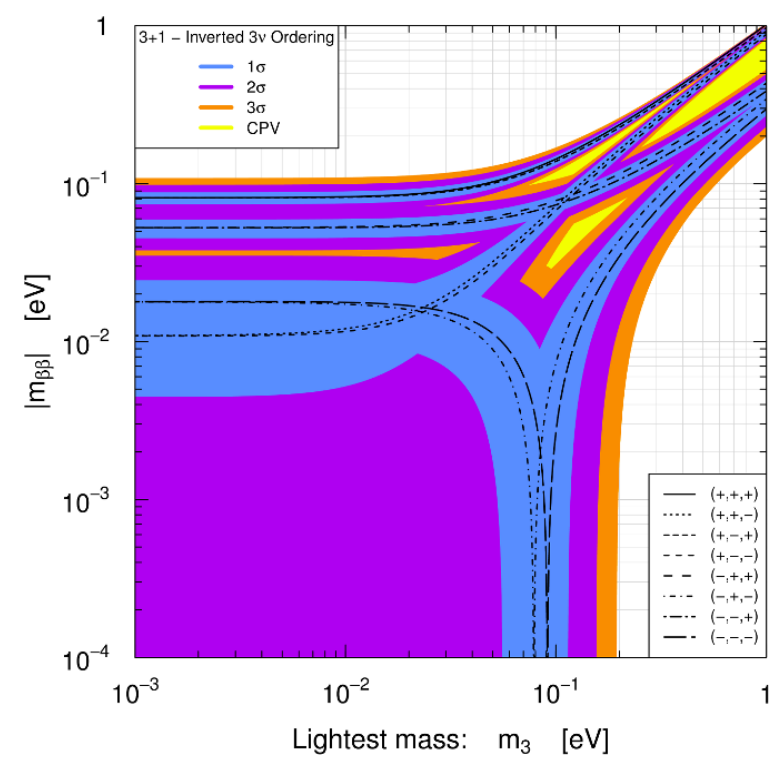

Figure 18. Value of the effective Majorana mass $\left|m_{\beta \beta}\right|$ as a function of the lightest neutrino mass $m_{3}$ in the case of $3+1$ mixing with Inverted Ordering of the three lightest neutrinos. The legend is explained in the caption of figure 12 .

\begin{tabular}{|ccccc|}
\hline & b.f. & $1 \sigma$ & $2 \sigma$ & $3 \sigma$ \\
\hline$m_{3}\left[10^{-2} \mathrm{eV}\right]$ & $<9.1$ & $<11.4$ & $<15.5$ & $<19.3$ \\
$m_{\beta}\left[10^{-2} \mathrm{eV}\right]$ & $4.8-10.3$ & $4.8-14.2$ & $4.7-17.6$ & $4.6-22.5$ \\
$\Sigma\left[10^{-2} \mathrm{eV}\right]$ & $9.8-29.9$ & $9.7-41.8$ & $9.5-52.3$ & $9.4-67.1$ \\
\hline
\end{tabular}

Table 3. Ranges of $m_{3}, m_{\beta}$ and $\Sigma$ for which there can be a complete cancellation of the four partial mass contributions to $\left|m_{\beta \beta}\right|$ for the best-fit values (b.f.) of the oscillation parameters and at $1 \sigma, 2 \sigma$ and $3 \sigma$ in the case of $3+1$ mixing with Inverted Ordering of the three lightest neutrinos.

\section{Conclusions}

We have presented accurate calculations of the effective Majorana mass $\left|m_{\beta \beta}\right|$ in neutrinoless double- $\beta$ decay in the standard case of $3 \nu$ mixing and in the case of $3+1$ neutrino mixing indicated by the reactor, Gallium and LSND anomalies (see refs. [18, 19]). We have taken into account the uncertainties of the standard $3 \nu$ mixing parameters obtained in the global fit of solar, atmospheric and long-baseline reactor and accelerator neutrino oscillation data presented in ref. [3] and the uncertainties on the additional mixing parameters in the $3+1$ case obtained from an update $[34,35]$ of the global fit of short-baseline neutrino oscillation data presented in ref. [19].

We have shown that the predictions for $\left|m_{\beta \beta}\right|$ in the cases of $3 \nu$ and $3+1$ mixing are quite different, in agreement with the previous discussions in refs. [13, 25-33]. Our paper improves these discussions by taking into account the uncertainties of all the mixing parameters and presenting all the results at $1 \sigma, 2 \sigma$ and $3 \sigma$. 


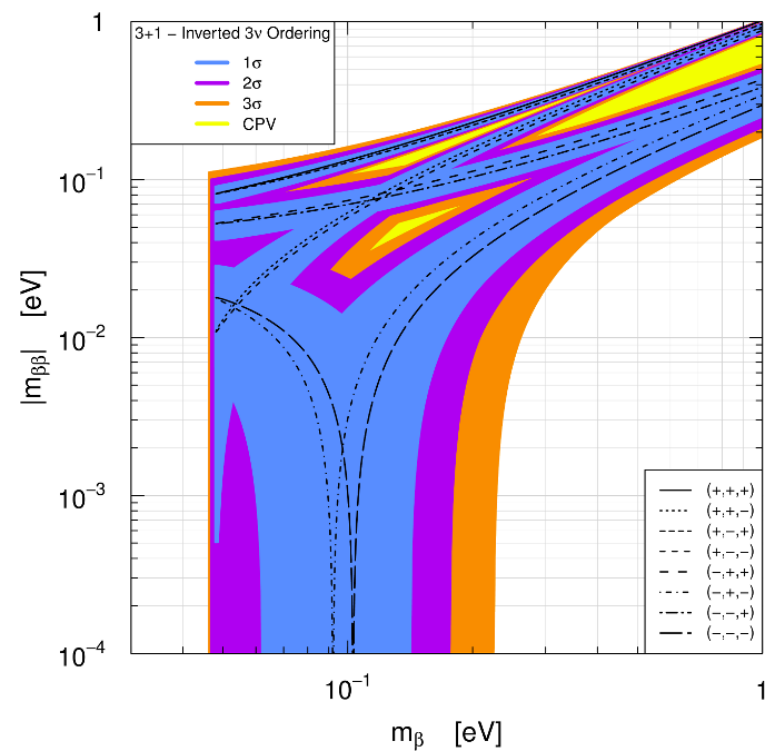

Figure 19. Value of the effective Majorana mass $\left|m_{\beta \beta}\right|$ as a function of sum of the three lightest neutrino masses $\Sigma$ in eq. (2.23) in the case of $3+1$ mixing with Inverted Ordering of the three lightest neutrinos. The legend is explained in the caption of figure 12 .

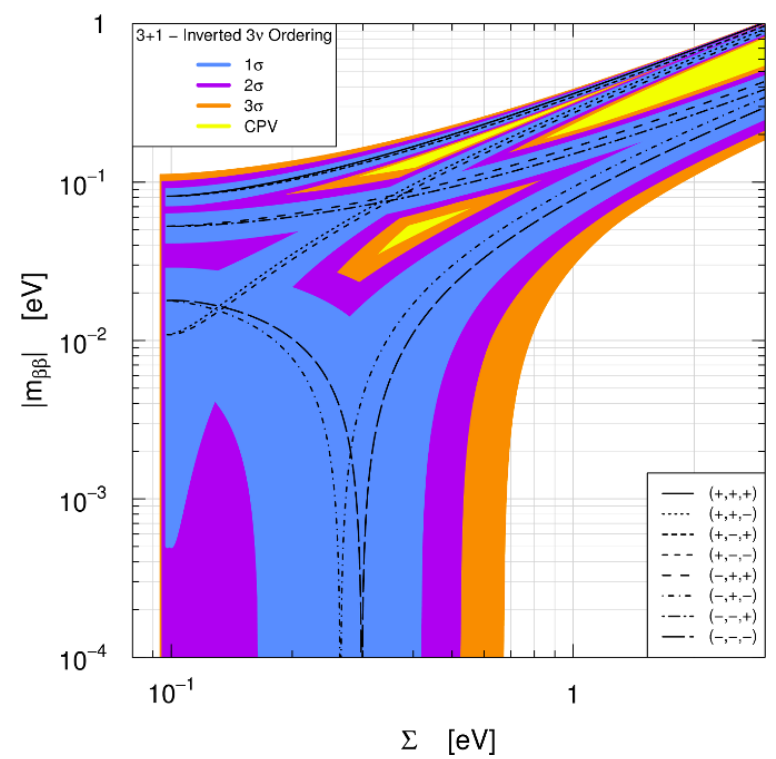

Figure 20. Value of the effective Majorana mass $\left|m_{\beta \beta}\right|$ as a function of effective electron neutrino mass $m_{\beta}$ in eq. (2.22) in the case of $3+1$ mixing with Inverted Ordering of the three lightest neutrinos. The legend is explained in the caption of figure 12 . 


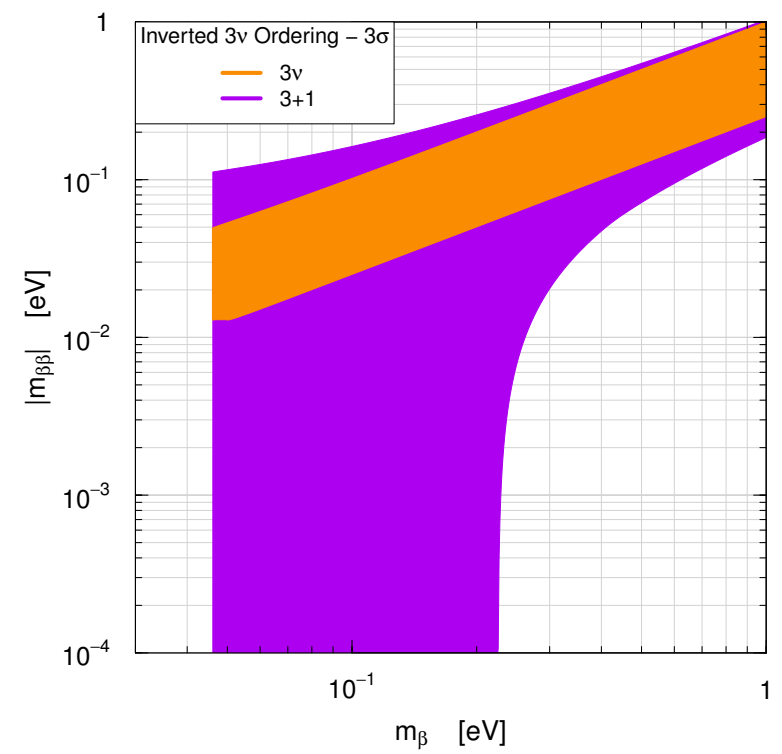

Figure 21. Comparison of the $3 \sigma$ allowed regions in the $m_{\beta}-\left|m_{\beta \beta}\right|$ plane in the cases of $3 \nu$ and $3+1$ mixing with Inverted Ordering of the three lightest neutrinos.

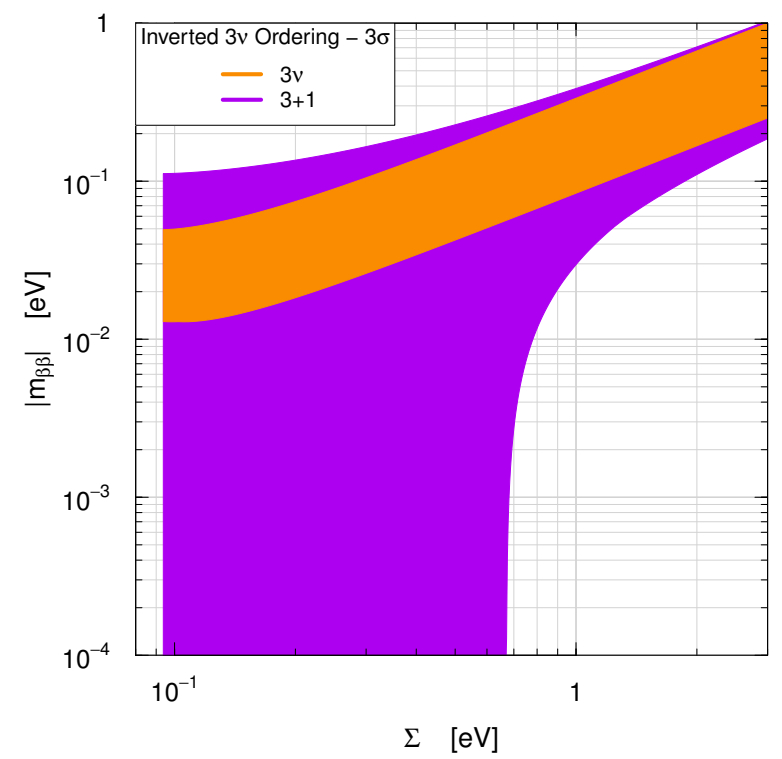

Figure 22. Comparison of the $3 \sigma$ allowed regions in the $\Sigma-\left|m_{\beta \beta}\right|$ plane in the cases of $3 \nu$ and $3+1$ mixing with Inverted Ordering of the three lightest neutrinos. 
We have presented accurate comparisons of the allowed regions in the planes $m_{\beta^{-}}$ $\left|m_{\beta \beta}\right|$ and $\Sigma-\left|m_{\beta \beta}\right|$ of measurable quantities, taking into account the two possibilities of Normal and Inverted Ordering of the three light lightest neutrinos. We have shown that future measurements of these quantities may distinguish the $3 \nu$ and $3+1$ cases if the mass ordering is determined by oscillation experiments (see refs. [1, 2]).

We have also introduced in section 2.1 a relatively simple method to determine the minimum value of $\left|m_{\beta \beta}\right|$ in the general case of $N$-neutrino mixing.

\section{Acknowledgments}

E.Z. thanks the support of funding grants 2013/02518-7 and 2014/23980-3, São Paulo Research Foundation (FAPESP). The work of C. Giunti is partially supported by the research grant Theoretical Astroparticle Physics number 2012CPPYP7 under the program PRIN 2012 funded by the Ministero dell'Istruzione, Università e della Ricerca (MIUR).

Open Access. This article is distributed under the terms of the Creative Commons Attribution License (CC-BY 4.0), which permits any use, distribution and reproduction in any medium, provided the original author(s) and source are credited.

\section{References}

[1] G. Bellini, L. Ludhova, G. Ranucci and F.L. Villante, Neutrino oscillations, Adv. High Energy Phys. 2014 (2014) 191960 [arXiv:1310.7858] [INSPIRE].

[2] Y. Wang and Z. zhong Xing, Neutrino Masses and Flavor Oscillations, arXiv:1504.06155 [INSPIRE].

[3] F. Capozzi, G.L. Fogli, E. Lisi, A. Marrone, D. Montanino and A. Palazzo, Status of three-neutrino oscillation parameters, circa 2013, Phys. Rev. D 89 (2014) 093018 [arXiv: 1312.2878] [INSPIRE].

[4] D.V. Forero, M. Tortola and J.W.F. Valle, Neutrino oscillations refitted, Phys. Rev. D 90 (2014) 093006 [arXiv: 1405.7540] [INSPIRE].

[5] M.C. Gonzalez-Garcia, M. Maltoni and T. Schwetz, Updated fit to three neutrino mixing: status of leptonic CP-violation, JHEP 11 (2014) 052 [arXiv: 1409.5439] [INSPIRE].

[6] G. Mention et al., The Reactor Antineutrino Anomaly, Phys. Rev. D 83 (2011) 073006 [arXiv: 1101.2755] [INSPIRE].

[7] T.A. Mueller et al., Improved Predictions of Reactor Antineutrino Spectra, Phys. Rev. C 83 (2011) 054615 [arXiv:1101.2663] [INSPIRE].

[8] P. Huber, On the determination of anti-neutrino spectra from nuclear reactors, Phys. Rev. C 84 (2011) 024617 [Erratum ibid. C 85 (2012) 029901] [arXiv:1106.0687] [INSPIRE].

[9] J.N. Abdurashitov et al., Measurement of the response of a Ga solar neutrino experiment to neutrinos from an Ar-37 source, Phys. Rev. C 73 (2006) 045805 [nucl-ex/0512041] [INSPIRE].

[10] M. Laveder, Unbound neutrino roadmaps, Nucl. Phys. Proc. Suppl. 168 (2007) 344 [inSPIRE].

[11] C. Giunti and M. Laveder, Short-Baseline Active-Sterile Neutrino Oscillations?, Mod. Phys. Lett. A 22 (2007) 2499 [hep-ph/0610352] [INSPIRE]. 
[12] C. Giunti and M. Laveder, Statistical Significance of the Gallium Anomaly, Phys. Rev. C 83 (2011) 065504 [arXiv: 1006 .3244] [INSPIRE].

[13] C. Giunti, M. Laveder, Y.F. Li, Q.Y. Liu and H.W. Long, Update of Short-Baseline Electron Neutrino and Antineutrino Disappearance, Phys. Rev. D 86 (2012) 113014 [arXiv: 1210.5715] [INSPIRE].

[14] F. Kaether, W. Hampel, G. Heusser, J. Kiko and T. Kirsten, Reanalysis of the GALLEX solar neutrino flux and source experiments, Phys. Lett. B 685 (2010) 47 [arXiv:1001.2731] [INSPIRE].

[15] SAGE collaboration, J.N. Abdurashitov et al., Measurement of the solar neutrino capture rate with gallium metal. III: Results for the 2002-2007 data-taking period, Phys. Rev. C 80 (2009) 015807 [arXiv:0901.2200] [INSPIRE].

[16] LSND collaboration, C. Athanassopoulos et al., Candidate events in a search for anti-muon-neutrino $\rightarrow$ anti-electron-neutrino oscillations, Phys. Rev. Lett. 75 (1995) 2650 [nucl-ex/9504002] [INSPIRE].

[17] LSND collaboration, A. Aguilar-Arevalo et al., Evidence for neutrino oscillations from the observation of anti-neutrino(electron) appearance in a anti-neutrino(muon) beam, Phys. Rev. D 64 (2001) 112007 [hep-ex/0104049] [INSPIRE].

[18] J. Kopp, P.A.N. Machado, M. Maltoni and T. Schwetz, Sterile neutrino oscillations: the global picture, JHEP 05 (2013) 050 [arXiv: 1303.3011] [INSPIRE].

[19] C. Giunti, M. Laveder, Y.F. Li and H.W. Long, Pragmatic View of Short-Baseline Neutrino Oscillations, Phys. Rev. D 88 (2013) 073008 [arXiv: 1308.5288] [InSPIRE].

[20] Particle Data Group collaboration, K. Olive et al., Review of Particle Physics, Chin. Phys. C 38 (2014) 090001 [inSPIRE].

[21] B. Pontecorvo, Neutrino Experiments and the Problem of Conservation of Leptonic Charge, Sov. Phys. JETP 26 (1968) 984 [INSPIRE].

[22] S.M. Bilenky and C. Giunti, Neutrinoless Double-Beta Decay: a Probe of Physics Beyond the Standard Model, Int. J. Mod. Phys. A 30 (2015) 1530001 [arXiv:1411.4791] [inSPIRE].

[23] C. Kraus et al., Final results from phase II of the Mainz neutrino mass search in tritium beta decay, Eur. Phys. J. C 40 (2005) 447 [hep-ex/0412056] [INSPIRE].

[24] Troitsk collaboration, V.N. Aseev et al., An upper limit on electron antineutrino mass from Troitsk experiment, Phys. Rev. D 84 (2011) 112003 [arXiv:1108.5034] [INSPIRE].

[25] S. Goswami and W. Rodejohann, Constraining mass spectra with sterile neutrinos from neutrinoless double beta decay, tritium beta decay and cosmology, Phys. Rev. D 73 (2006) 113003 [hep-ph/0512234] [INSPIRE].

[26] S. Goswami and W. Rodejohann, MiniBooNE results and neutrino schemes with 2 sterile neutrinos: Possible mass orderings and observables related to neutrino masses, JHEP 10 (2007) 073 [arXiv:0706.1462] [INSPIRE].

[27] J. Barry, W. Rodejohann and H. Zhang, Light Sterile Neutrinos: Models and Phenomenology, JHEP 07 (2011) 091 [arXiv: 1105.3911] [INSPIRE].

[28] Y.F. Li and S.-s. Liu, Vanishing effective mass of the neutrinoless double beta decay including light sterile neutrinos, Phys. Lett. B 706 (2012) 406 [arXiv:1110.5795] [INSPIRE].

[29] W. Rodejohann, Neutrinoless double beta decay and neutrino physics, J. Phys. G 39 (2012) 124008 [arXiv: 1206.2560] [INSPIRE]. 
[30] I. Girardi, A. Meroni and S.T. Petcov, Neutrinoless double beta decay in the presence of light sterile neutrinos, JHEP 11 (2013) 146 [arXiv:1308.5802] [INSPIRE].

[31] S. Pascoli, M. Mitra and S. Wong, Effect of cancellation in neutrinoless double beta decay, Phys. Rev. D 90 (2014) 093005 [arXiv: 1310.6218] [INSPIRE].

[32] A. Meroni and E. Peinado, The quest for neutrinoless double beta decay: Pseudo-Dirac, Majorana and sterile neutrinos, Phys. Rev. D 90 (2014) 053002 [arXiv:1406.3990] [INSPIRE].

[33] A. Abada, V. De Romeri and A.M. Teixeira, Effect of steriles states on lepton magnetic moments and neutrinoless double beta decay, JHEP 09 (2014) 074 [arXiv:1406.6978] [INSPIRE].

[34] C. Giunti, Global Status of Sterile Neutrino Scenarios, talk presented at NeuTel 2015, XVI International Workshop on Neutrino Telescopes, 2-6 March 2015, Venice, Italy.

[35] S. Gariazzo, C. Giunti, M. Laveder, Y.F. Li and E.M. Zavanin, Light sterile neutrinos, arXiv: 1507.08204.

[36] J.J. Gomez-Cadenas, J. Martin-Albo, M. Mezzetto, F. Monrabal and M. Sorel, The Search for neutrinoless double beta decay, Riv. Nuovo Cim. 35 (2012) 29 [arXiv:1109.5515] [INSPIRE].

[37] A. Giuliani and A. Poves, Neutrinoless Double-Beta Decay, Adv. High Energy Phys. 2012 (2012) 857016 .

[38] B. Schwingenheuer, Status and prospects of searches for neutrinoless double beta decay, Annalen Phys. 525 (2013) 269 [arXiv:1210.7432] [INSPIRE].

[39] O. Cremonesi and M. Pavan, Challenges in Double Beta Decay, arXiv:1310.4692 [INSPIRE].

[40] CUORE collaboration, D.R. Artusa et al., Exploring the Neutrinoless Double Beta Decay in the Inverted Neutrino Hierarchy with Bolometric Detectors, Eur. Phys. J. C 74 (2014) 3096 [arXiv: 1404.4469] [INSPIRE].

[41] J.J. Gómez-Cadenas and J. Martín-Albo, Phenomenology of neutrinoless double beta decay, PoS GSSI14 (2015) 004 [arXiv: 1502.00581] [inSPIRE].

[42] A. Faessler and F. Simkovic, Double beta decay, J. Phys. G 24 (1998) 2139 [hep-ph/9901215] [INSPIRE].

[43] K.-w. Choi, K.S. Jeong and W.Y. Song, Operator analysis of neutrinoless double beta decay, Phys. Rev. D 66 (2002) 093007 [hep-ph/0207180] [INSPIRE].

[44] A. Ibarra, E. Molinaro and S.T. Petcov, TeV Scale See-Saw Mechanisms of Neutrino Mass Generation, the Majorana Nature of the Heavy Singlet Neutrinos and $(\beta \beta)_{-} 0 \nu$-Decay, JHEP 09 (2010) 108 [arXiv: 1007.2378] [INSPIRE].

[45] V. Tello, M. Nemevšek, F. Nesti, G. Senjanović and F. Vissani, Left-Right Symmetry: from LHC to Neutrinoless Double Beta Decay, Phys. Rev. Lett. 106 (2011) 151801 [arXiv: 1011.3522] [INSPIRE].

[46] W. Rodejohann, Neutrino-less Double Beta Decay and Particle Physics, Int. J. Mod. Phys. E 20 (2011) 1833 [arXiv:1106.1334] [INSPIRE].

[47] F. del Aguila, A. Aparici, S. Bhattacharya, A. Santamaria and J. Wudka, Effective Lagrangian approach to neutrinoless double beta decay and neutrino masses, JHEP 06 (2012) 146 [arXiv: 1204.5986] [INSPIRE]. 
[48] F.F. Deppisch, M. Hirsch and H. Pas, Neutrinoless Double Beta Decay and Physics Beyond the Standard Model, J. Phys. G 39 (2012) 124007 [arXiv:1208.0727] [InSPIRE].

[49] A. de Gouvêa and P. Vogel, Lepton Flavor and Number Conservation and Physics Beyond the Standard Model, Prog. Part. Nucl. Phys. 71 (2013) 75 [arXiv:1303.4097] [InSPIRE].

[50] S.M. Bilenky, S. Pascoli and S.T. Petcov, Majorana neutrinos, neutrino mass spectrum, CP-violation and neutrinoless double beta decay. 1. The Three neutrino mixing case, Phys. Rev. D 64 (2001) 053010 [hep-ph/0102265] [INSPIRE].

[51] S. Pascoli, S.T. Petcov and W. Rodejohann, On the CP-violation associated with Majorana neutrinos and neutrinoless double beta decay, Phys. Lett. B 549 (2002) 177 [hep-ph/0209059] [INSPIRE].

[52] A. Joniec and M. Zralek, Conditions for detecting CP-violation via neutrinoless double beta decay, Phys. Rev. D 73 (2006) 033001 [hep-ph/0411070] [INSPIRE].

[53] S. Pascoli, S.T. Petcov and T. Schwetz, The Absolute neutrino mass scale, neutrino mass spectrum, Majorana CP-violation and neutrinoless double-beta decay, Nucl. Phys. B 734 (2006) 24 [hep-ph/0505226] [INSPIRE].

[54] F. Simkovic, S.M. Bilenky, A. Faessler and T. Gutsche, Possibility of measuring the $C P$ Majorana phases in $0 \nu \beta \beta$ decay, Phys. Rev. D 87 (2013) 073002 [arXiv:1210.1306] [INSPIRE].

[55] A. de Gouvêa, B. Kayser and R.N. Mohapatra, Manifest CP-violation from Majorana phases, Phys. Rev. D 67 (2003) 053004 [hep-ph/0211394] [INSPIRE].

[56] KamLAND-Zen collaboration, A. Gando et al., Limit on Neutrinoless $\beta \beta$ Decay of ${ }^{136}$ Xe from the First Phase of KamLAND-Zen and Comparison with the Positive Claim in ${ }^{76} G e$, Phys. Rev. Lett. 110 (2013) 062502 [arXiv:1211.3863] [INSPIRE].

[57] H.V. Klapdor-Kleingrothaus et al., Latest results from the Heidelberg-Moscow double beta decay experiment, Eur. Phys. J. A 12 (2001) 147 [hep-ph/0103062] [INSPIRE].

[58] IGEX collaboration, C.E. Aalseth et al., The IGEX Ge-76 neutrinoless double beta decay experiment: Prospects for next generation experiments, Phys. Rev. D 65 (2002) 092007 [hep-ex/0202026] [INSPIRE].

[59] GERDA collaboration, M. Agostini et al., Results on Neutrinoless Double- $\beta$ Decay of ${ }^{76}$ Ge from Phase I of the GERDA Experiment, Phys. Rev. Lett. 111 (2013) 122503 [arXiv: 1307.4720] [INSPIRE].

[60] NEMO-3 collaboration, R. Arnold et al., Search for neutrinoless double-beta decay of ${ }^{100}$ Mo with the NEMO-3 detector, Phys. Rev. D 89 (2014) 111101 [arXiv:1311.5695] [INSPIRE].

[61] E. Andreotti et al., 130Te Neutrinoless Double-Beta Decay with CUORICINO, Astropart. Phys. 34 (2011) 822 [arXiv: 1012.3266] [INSPIRE].

[62] EXO-200 collaboration, J.B. Albert et al., Search for Majorana neutrinos with the first two years of EXO-200 data, Nature $\mathbf{5 1 0}$ (2014) 229 [arXiv:1402.6956] [INSPIRE].

[63] F. Vissani, Signal of neutrinoless double beta decay, neutrino spectrum and oscillation scenarios, JHEP 06 (1999) 022 [hep-ph/9906525] [INSPIRE].

[64] Z.-z. Xing and Y.-L. Zhou, Geometry of the effective Majorana neutrino mass in the $0 \nu \beta \beta$ decay, Chin. Phys. C 39 (2015) 011001 [arXiv:1404.7001] [INSPIRE].

[65] Planck collaboration, P.A.R. Ade et al., Planck 2015 results. XIII. Cosmological parameters, arXiv:1502.01589 [INSPIRE]. 
[66] V.D. Barger and K. Whisnant, Majorana neutrino masses from neutrinoless double beta decay and cosmology, Phys. Lett. B 456 (1999) 194 [hep-ph/9904281] [INSPIRE].

[67] K. Matsuda, N. Takeda, T. Fukuyama and H. Nishiura, MNS parameters from neutrino oscillations, single beta decay and double beta decay, Phys. Rev. D 64 (2001) 013001 [hep-ph/0012357] [INSPIRE].

[68] V. Barger, S.L. Glashow, D. Marfatia and K. Whisnant, Neutrinoless double beta decay can constrain neutrino dark matter, Phys. Lett. B 532 (2002) 15 [hep-ph/0201262] [INSPIRE].

[69] G.L. Fogli et al., Observables sensitive to absolute neutrino masses: Constraints and correlations from world neutrino data, Phys. Rev. D 70 (2004) 113003 [hep-ph/0408045] [INSPIRE].

[70] C. Giunti and C.W. Kim, Fundamentals of Neutrino Physics and Astrophysics, Oxford University Press, Oxford U.K. (2007).

[71] V. Barger, S.L. Glashow, P. Langacker and D. Marfatia, No go for detecting CP-violation via neutrinoless double beta decay, Phys. Lett. B 540 (2002) 247 [hep-ph/0205290] [INSPIRE].

[72] H. Nunokawa, W.J.C. Teves and R. Zukanovich Funchal, Constraining the absolute neutrino mass scale and Majorana CP-violating phases by future 0 neutrino beta beta decay experiments, Phys. Rev. D 66 (2002) 093010 [hep-ph/0206137] [InSPIRE].

[73] H. Minakata, H. Nunokawa and A.A. Quiroga, Constraining Majorana CP phase in the precision era of cosmology and the double beta decay experiment, PTEP 2015 (2015) 033B03 [arXiv: 1402.6014] [INSPIRE].

[74] S. Dell'Oro, S. Marcocci and F. Vissani, New expectations and uncertainties on neutrinoless double beta decay, Phys. Rev. D 90 (2014) 033005 [arXiv:1404.2616] [INSPIRE].

[75] J.D. Vergados, H. Ejiri and F. Simkovic, Theory of Neutrinoless Double Beta Decay, Rept. Prog. Phys. 75 (2012) 106301 [arXiv: 1205.0649] [INSPIRE].

[76] J. Suhonen, Double beta decay: An interface between nuclear, particle and atomic physics, J. Phys. Conf. Ser. 413 (2013) 012016 [InSPIRE].

[77] N. Yoshida and F. Iachello, Two neutrino double- $\beta$ decay in the interacting boson-fermion model, PTEP 2013 (2013) 043D01 [arXiv:1301.7172] [INSPIRE].

[78] KATRIN collaboration, S. Mertens, Status of the KATRIN Experiment and Prospects to Search for keV-mass Sterile Neutrinos in Tritium $\beta$-decay, Phys. Procedia 61 (2015) 267.

[79] R.E. Shrock, New Tests For and Bounds On, Neutrino Masses and Lepton Mixing, Phys. Lett. B 96 (1980) 159 [INSPIRE].

[80] B.H.J. McKellar, The Influence of Mixing of Finite Mass Neutrinos on Beta Decay Spectra, Phys. Lett. B 97 (1980) 93 [INSPIRE].

[81] I. Yu. Kobzarev, B.V. Martemyanov, L.B. Okun and M.G. Shchepkin, The phenomenology of neutrino oscillations, Sov. J. Nucl. Phys. 32 (1980) 823 [INSPIRE].

[82] F. Vissani, Nonoscillation searches of neutrino mass in the age of oscillations, Nucl. Phys. Proc. Suppl. 100 (2001) 273 [hep-ph/0012018] [INSPIRE].

[83] Y. Farzan and A. Yu. Smirnov, On the effective mass of the electron neutrino in beta decay, Phys. Lett. B 557 (2003) 224 [hep-ph/0211341] [INSPIRE].

[84] Y.-Z. Chu and M. Cirelli, Sterile neutrinos, lepton asymmetries, primordial elements: How much of each?, Phys. Rev. D 74 (2006) 085015 [astro-ph/0608206] [INSPIRE]. 
[85] S. Hannestad, I. Tamborra and T. Tram, Thermalisation of light sterile neutrinos in the early universe, JCAP 07 (2012) 025 [arXiv: 1204.5861] [INSPIRE].

[86] A. Mirizzi, N. Saviano, G. Miele and P.D. Serpico, Light sterile neutrino production in the early universe with dynamical neutrino asymmetries, Phys. Rev. D 86 (2012) 053009 [arXiv: 1206.1046] [INSPIRE].

[87] N. Saviano et al., Multi-momentum and multi-flavour active-sterile neutrino oscillations in the early universe: role of neutrino asymmetries and effects on nucleosynthesis, Phys. Rev. D 87 (2013) 073006 [arXiv: 1302.1200] [INSPIRE].

[88] S. Hannestad, R.S. Hansen and T. Tram, Can active-sterile neutrino oscillations lead to chaotic behavior of the cosmological lepton asymmetry?, JCAP 04 (2013) 032 [arXiv: 1302.7279] [INSPIRE].

[89] S. Hannestad, R.S. Hansen and T. Tram, How Self-Interactions can Reconcile Sterile Neutrinos with Cosmology, Phys. Rev. Lett. 112 (2014) 031802 [arXiv:1310.5926] [INSPIRE].

[90] B. Dasgupta and J. Kopp, Cosmologically Safe eV-Scale Sterile Neutrinos and Improved Dark Matter Structure, Phys. Rev. Lett. 112 (2014) 031803 [arXiv:1310.6337] [INSPIRE].

[91] T. Bringmann, J. Hasenkamp and J. Kersten, Tight bonds between sterile neutrinos and dark matter, JCAP 07 (2014) 042 [arXiv: 1312 .4947] [inSPIRE].

[92] P. Ko and Y. Tang, $\nu \Lambda M D M$ : A Model for Sterile Neutrino and Dark Matter Reconciles Cosmological and Neutrino Oscillation Data after BICEP2, Phys. Lett. B 739 (2014) 62 [arXiv: 1404.0236] [INSPIRE].

[93] M. Archidiacono, S. Hannestad, R.S. Hansen and T. Tram, Cosmology with self-interacting sterile neutrinos and dark matter - A pseudoscalar model, Phys. Rev. D 91 (2015) 065021 [arXiv: 1404.5915] [INSPIRE].

[94] N. Saviano, O. Pisanti, G. Mangano and A. Mirizzi, Unveiling secret interactions among sterile neutrinos with big-bang nucleosynthesis, Phys. Rev. D 90 (2014) 113009 [arXiv: 1409.1680] [INSPIRE].

[95] A. Mirizzi, G. Mangano, O. Pisanti and N. Saviano, Collisional production of sterile neutrinos via secret interactions and cosmological implications, Phys. Rev. D 91 (2015) 025019 [arXiv: 1410.1385] [INSPIRE].

[96] T. Rehagen and G.B. Gelmini, Effects of kination and scalar-tensor cosmologies on sterile neutrinos, JCAP 06 (2014) 044 [arXiv: 1402.0607] [INSPIRE].

[97] C.M. Ho and R.J. Scherrer, Sterile Neutrinos and Light Dark Matter Save Each Other, Phys. Rev. D 87 (2013) 065016 [arXiv: 1212.1689] [INSPIRE].

[98] KATRIN collaboration, R.G.H. Robertson, KATRIN: an experiment to determine the neutrino mass from the beta decay of tritium, arXiv:1307.5486 [INSPIRE].

[99] B. Audren, J. Lesgourgues, S. Bird, M.G. Haehnelt and M. Viel, Neutrino masses and cosmological parameters from a Euclid-like survey: Markov Chain Monte Carlo forecasts including theoretical errors, JCAP 01 (2013) 026 [arXiv:1210.2194] [INSPIRE]. 